\title{
HARD LEFSCHETZ THEOREM FOR NONRATIONAL POLYTOPES
}

\author{
KALLE KARU
}

\begin{abstract}
The Hard Lefschetz theorem is known to hold for the intersection cohomology of the toric variety associated to a rational convex polytope. One can construct the intersection cohomology combinatorially from the polytope, hence it is well defined even for nonrational polytopes when there is no variety associated to it. We prove the Hard Lefschetz theorem for the intersection cohomology of a general polytope.
\end{abstract}

\section{INTRODUCTION}

If $P$ is a simple convex $n$-dimensional polytope in $\mathbb{R}^{n}$, define

$$
h_{k}=\sum_{i \geq k} f_{i}(-1)^{i-k}\left(\begin{array}{l}
i \\
k
\end{array}\right),
$$

where $f_{i}$ is the number of $i$-dimensional faces of $P$. Then knowing the numbers $h_{k}$ is equivalent to knowing the numbers $f_{i}$. The following conditions are satisfied by the numbers $h_{k}$ :

(1) Dehn-Sommerville equations: $h_{i}=h_{n-i}$.

(2) Unimodality: $1=h_{0} \leq h_{1} \leq \ldots \leq h_{[n / 2]}$.

To prove the unimodality condition (in fact a stronger condition conjectured by P. McMullen), R. Stanley in [8] constructed a projective toric variety $X_{P}$ so that $h_{i}$ are the even Betti numbers of the (singular) cohomology of $X_{P}$. The two conditions then follow from the Poincaré duality and the Hard Lefschetz theorem for $X_{P}$. Later in [9] Stanley generalized the definition of the $h$-vector to an arbitrary polytope $P$ in such a way that if the polytope is rational the numbers $h_{i}$ are the intersection cohomology Betti numbers of the associated toric variety (see [4 for a validation of this claim). Stanley also proved that the Dehn-Sommerville equations hold for the generalized vector $h$. If the polytope $P$ is rational, the unimodality condition follows from the Hard Lefschetz theorem in intersection cohomology of the associated toric variety. The goal of this paper is to prove the Hard Lefschetz theorem for a general polytope, generalizing McMullen's purely combinatorial proof of the Hard Lefschetz theorem for a simple polytope [7].

Our proof is based on the description of the intersection cohomology of a fan (e.g., the normal fan of a polytope) given in [1, 2] . When the fan is simplicial, the torus-equivariant cohomology of the corresponding toric variety can be identified with the space of conewise polynomial functions on the fan. This set is naturally a module over the algebra $A$ of global polynomial functions. Taking the quotient by the ideal $I \subset A$ generated by global linear functions

The author was partially supported by NSF grant DMS-0070678. 
gives us the non-equivariant intersection cohomology. In order to generalize this, one views the conewise polynomial functions as global sections of a sheaf on the fan with respect to a certain topology. On a general fan $\Sigma$ one defines the equivariant intersection cohomology sheaf $\mathcal{L}_{\Sigma}$; the equivariant intersection cohomology of the fan $\Sigma$ is then the $A$-module of global sections of this sheaf. Taking the quotient by the ideal $I$, we obtain the non-equivariant intersection cohomology $I H(\Sigma)$ of the fan $\Sigma$.

If the fan $\Sigma$ is projective, there exists a conewise linear strictly convex function $l$ on $\Sigma$. Multiplication with this function defines a degree 2 homomorphism of the sheaf $\mathcal{L}$ (we assume that linear functions have degree 2), and it also induces a degree 2 map of $I H(\Sigma)$.

Theorem 0.1 (Hard Lefschetz). Let $\Sigma$ be a projective fan of dimension $n$ and let $l$ be a conewise linear strictly convex function on $\Sigma$. Then multiplication with $l$ defines a Lefschetz operation on $I H(\Sigma)$. That means

$$
l^{i}: I H^{n-i}(\Sigma) \longrightarrow I H^{n+i}(\Sigma)
$$

is an isomorphism for all $i>0$.

The theorem is known for simplicial fans [8, 7] and for rational fans [9] (a fan in $\mathbb{R}^{n}$ is rational if every cone is generated by a set of vectors with rational coordinates). V. Timorin [10] recently proved the Hard Lefschetz theorem for certain fans in which all cones of dimension less than $n$ are simplicial.

The Hard Lefschetz theorem follows from an even stronger statement, the Hodge-Riemann-Minkowski relations. Using the bilinear Poincaré duality pairing [1]:

$$
\begin{aligned}
I H^{n-i}(\Sigma) \times I H^{n+i}(\Sigma) & \longrightarrow \mathbb{R} \\
(x, y) & \longmapsto\langle x \cdot y\rangle,
\end{aligned}
$$

we define the quadratic form $Q_{l}(x)=\left\langle l^{i} x \cdot x\right\rangle$ on $I H^{n-i}(\Sigma)$. Then if

$$
I P^{n-i}(\Sigma)=\operatorname{ker} l^{i+1}: I H^{n-i}(\Sigma) \longrightarrow I H^{n+i+2}(\Sigma)
$$

is the primitive cohomology, we have

Theorem 0.2 (Hodge-Riemann-Minkowski bilinear relations). The quadratic form

$$
(-1)^{\frac{n-i}{2}} Q_{l}
$$

is positive definite on $I P^{n-i}(\Sigma)$ for all $i \geq 0$.

We remark that the intersection cohomology of a complete fan is nonzero only in even degrees, hence the power $\frac{n-i}{2}$ is always an integer. McMullen in [7] proved the Hodge-Riemann-Minkowski relations for simplicial fans (see also the proof by Timorin [11]). The names Hodge-Riemann refer to the usual HodgeRiemann bilinear relations for Kähler manifolds; the inequalities in degree $n-i=2$ reduce to the Minkowski inequalities on polytopes [7].

We will assume the Hodge-Riemann-Minkowski inequalities in the simplicial case and deduce Theorem 0.2 from it.

Acknowledgments. I would like to thank Gottfried Barthel, Valery Lunts and Günter Ziegler for helpful comments. The preprint in the current form owes much to the advice of Karl-Heinz Fieseler. Almost the entire last section 
has been rewritten following his suggestions. I take credit for any remaining errors.

\section{INTERSECTION COHOMOLOGY OF FANS}

We start by recalling the definition of the intersection cohomology of a fan. The main references here are [1, 2, 3].

Let $V$ be a real vector space of dimension $n$. We denote by $A$ the algebra of polynomial functions on $V$ :

$$
A=\operatorname{Sym} V^{*}
$$

graded so that linear functions have degree 2 . The ideal generated by linear functions in $A$ is denoted by $I$, and the quotient of an $A$-module $M$ by the submodule $I M$ is denoted by $\bar{M}$.

1.1. Cones and fans. A polyhedral cone $\sigma$ in $V$ is a cone generated by a finite set of vectors $v_{1}, \ldots v_{N} \in V$ :

$$
\sigma=\mathbb{R}_{\geq 0} v_{1}+\ldots+\mathbb{R}_{\geq 0} v_{N} .
$$

We only consider pointed cones, which means that $\{0\}$ is the largest sub-vector space in a cone. Given a cone $\sigma \subset V$ and a linear function $f$ on $V$ such that $f$ takes non-negative values on $\sigma$, the cone $\tau=\sigma \cap \operatorname{ker} f$ is called a face of $\sigma$. We denote this relation by $\tau \prec \sigma$. A face of $\operatorname{dimension} \operatorname{dim} \sigma-1$ is called a facet of $\sigma$.

A fan $\Sigma$ in $V$ is a finite collection of cones in $V$ such that (i) if $\sigma \in \Sigma$ and $\tau$ is a face of $\sigma$ then $\tau \in \Sigma$; and (ii) if $\sigma_{1}, \sigma_{2} \in \Sigma$ then the intersection $\sigma_{1} \cap \sigma_{2}$ is a face of both $\sigma_{1}$ and $\sigma_{2}$. Examples of fans include the fan $[\sigma]$ consisting of all faces of a cone $\sigma$, and the fan $\partial \sigma=[\sigma]-\{\sigma\}$. Given a cone $\sigma \in \Sigma$ we define

$$
\begin{aligned}
& \operatorname{Star}_{\Sigma}(\sigma)=\{\delta \in \Sigma \mid \sigma \prec \delta\}, \\
& \overline{\operatorname{Star}}_{\Sigma}(\sigma)=\left\{\tau \in \Sigma \mid \tau \prec \delta \text { for some } \delta \in \operatorname{Star}_{\Sigma}(\sigma)\right\}, \\
& \operatorname{Link}_{\Sigma}(\sigma)=\left\{\nu \in \overline{\operatorname{Star}}_{\Sigma}(\sigma) \mid \nu \cap \sigma=\{0\}\right\} .
\end{aligned}
$$

Then $\overline{\operatorname{Star}}_{\Sigma}(\sigma)$ and $\operatorname{Link}_{\Sigma}(\sigma)$ are subfans of $\Sigma$.

The support of a fan $\Sigma$ is $|\Sigma|=\cup_{\sigma \in \Sigma} \sigma$. A fan is complete if its support is $V$. The relative interior of a cone $\sigma$ is $\sigma-|\partial \sigma|$.

A cone $\sigma$ is called simplicial if it can be generated by $\operatorname{dim} \sigma$ vectors. A fan is simplicial if all its cones are simplicial.

1.2. Sheaves on a fan. We consider a fan $\Sigma$ as a set of cones and define a topology on it so that open sets are the subfans of $\Sigma$. We can then consider sheaves of $\mathbb{R}$-vector spaces on $\Sigma$ with respect to this topology. To give a sheaf $\mathcal{F}$ on $\Sigma$, it suffices to specify for each cone $\sigma \in \Sigma$ the stalk $\mathcal{F}_{\sigma}=\mathcal{F}([\sigma])$ and the restriction map $\mathcal{F}_{\sigma} \rightarrow \mathcal{F}(\partial \sigma)$.

The structure sheaf $\mathcal{A}_{\Sigma}$ is defined by setting $\mathcal{A}_{\Sigma, \sigma}=\operatorname{Sym}(\operatorname{Span} \sigma)^{*}$, the space of polynomial functions on $\sigma$; the restriction map $\mathcal{A}_{\Sigma, \sigma} \rightarrow \mathcal{A}_{\Sigma}(\partial \sigma)$ is defined by restriction of functions. Then $\mathcal{A}_{\Sigma}$ is a sheaf of algebras, naturally graded by degree (again, conewise linear functions have degree 2). Multiplication with elements of $A$ gives $\mathcal{A}_{\Sigma}$ the structure of a sheaf of $A$-modules. 
An equivariant intersection cohomology sheaf $\mathcal{L}_{\Sigma}$ of $\Sigma$ is a sheaf of $\mathcal{A}_{\Sigma^{-}}$ modules satisfying the following properties:

(1) Normalization: $\mathcal{L}_{\Sigma, 0}=\mathbb{R}$.

(2) Local freeness: $\mathcal{L}_{\Sigma, \sigma}$ is a free $\mathcal{A}_{\Sigma, \sigma}$-module for any $\sigma \in \Sigma$.

(3) Minimal flabbiness: Modulo the ideal $I \subset A$ the restriction map induces an isomorphism

$$
\overline{\mathcal{L}}_{\Sigma, \sigma} \longrightarrow \overline{\mathcal{L}_{\Sigma}(\partial \sigma)}
$$

Note that the third property of an equivariant intersection cohomology sheaf implies that $\mathcal{L}_{\Sigma}$ is flabby: the restriction map $\mathcal{L}_{\Sigma}(U) \rightarrow \mathcal{L}_{\Sigma}(V)$ is surjective for any open sets $V \subset U \subset \Sigma$. Equivariant intersection cohomology sheaves exist for any fan $\Sigma$, and any two of them are isomorphic, hence we may call a sheaf of $\mathcal{A}_{\Sigma}$-modules satisfying the three properties the equivariant intersection cohomology sheaf. As a special case, $\mathcal{L}_{\Sigma} \simeq \mathcal{A}_{\Sigma}$ if and only if the fan $\Sigma$ is simplicial.

One can construct the sheaf $\mathcal{L}_{\Sigma}$ by induction on the dimension of cones as follows: assume that $\mathcal{L}_{\Sigma}$ is defined on $\partial \sigma$, and put

$$
\mathcal{L}_{\Sigma, \sigma}=\overline{\mathcal{L}_{\Sigma}(\partial \sigma)} \otimes \mathcal{A}_{\Sigma, \sigma}
$$

with the obvious restriction maps. (Note! All tensor products are taken over $\mathbb{R}$ unless specified otherwise.) From this construction it is clear that if $\Delta \subset$ $\Sigma$ is a subfan, then $\left.\mathcal{L}_{\Delta} \simeq \mathcal{L}_{\Sigma}\right|_{\Delta}$. To simplify notation, we will often omit the subscript $\Sigma$ and call the equivariant intersection cohomology sheaf simply $\mathcal{L}$. For example, the stalk $\mathcal{L}_{\sigma}$ for a cone $\sigma$ is defined up to isomorphism independent of the fan containing $\sigma$. We use similar omission of the subscript for the structure sheaf $\mathcal{A}_{\Sigma}$. Then $\mathcal{A}_{\sigma}=\mathcal{A}_{\Sigma, \sigma}$ is the algebra of polynomial functions on $\sigma$.

The (non-equivariant) intersection cohomology of a fan $\Sigma$ is defined to be the $A$-module of global sections of the equivariant intersection cohomology sheaf modulo the ideal $I$ :

$$
I H(\Sigma)=\overline{\mathcal{L}(\Sigma)}
$$

Since the equivariant intersection cohomology sheaf is a graded $A$-module and $I$ is a graded ideal, $I H(\Sigma)$ inherits a natural grading.

1.3. Morphisms of fans. Let $\Sigma$ be a fan in $V$ and $\Delta$ a fan in $W$. A conewise linear morphism $\Sigma \rightarrow \Delta$ is a pair $(|\phi|, \phi)$, where $|\phi|:|\Sigma| \rightarrow|\Delta|$ maps each cone $\sigma \in \Sigma$ linearly to some cone $\delta \in \Delta$, and $\phi$ is the induced continuous map between the fan spaces. We often denote the pair $(|\phi|, \phi)$ simply by $\phi$. Note that we do not require the map $|\phi|$ to be the restriction of a linear map $V \rightarrow W$; rather, the map $|\phi|$ is linear only when restricted to a cone.

If $\phi: \Sigma \rightarrow \Delta$ is a conewise linear morphism then by composing a section of $\mathcal{A}_{\Delta}$ with $|\phi|$, we get a section of $\mathcal{A}_{\Sigma}$, hence $\phi$ defines a morphism of ringed spaces. If $\mathcal{G}$ is a sheaf of $\mathcal{A}_{\Sigma}$-modules then $\phi_{*} \mathcal{G}$ has the structure of an $\mathcal{A}_{\Delta^{-}}$ module. For a sheaf $\mathcal{F}$ on $\Delta$ we denote the pullback sheaf by $\phi^{-1} \mathcal{F}$. If, moreover, $\mathcal{F}$ is a sheaf of $\mathcal{A}_{\Delta}$-modules, we define the sheaf of $\mathcal{A}_{\Sigma}$-modules

$$
\phi^{*} \mathcal{F}=\phi^{-1} \mathcal{F} \otimes_{\phi^{-1}} \mathcal{A}_{\Delta} \mathcal{A}_{\Sigma} .
$$


As an example, suppose $\phi: \Sigma \rightarrow \Delta$ is a conewise linear isomorphism. Then since $|\phi|$ maps every cone $\sigma \in \Sigma$ isomorphically onto some cone $\delta \in \Delta$, it follows that $\phi_{*} \mathcal{L}_{\Sigma} \simeq \mathcal{L}_{\Delta}$. In particular, we get an isomorphism of the vector spaces of global sections $\mathcal{L}(\Sigma) \simeq \mathcal{L}(\Delta)$; however, the $A$-module structures may be very different and the intersection cohomologies of the two fans need not be isomorphic. If we know that the spaces of global sections are finitely generated free $A$-modules (for example, when $\Sigma$ and $\Delta$ are complete), then by counting the dimensions of the graded pieces, we get a non-canonical isomorphism between the intersection cohomologies.

1.4. Subdivisions of fans. A conewise linear morphism of fans $s: \Sigma \rightarrow \Delta$ in the same space $V$ is called a subdivision if $|\Sigma|=|\Delta|$ and $|s|$ is the identity map. It follows from the decomposition theorem [1, 2] that if $s$ is a subdivision then $\mathcal{L}_{\Delta}$ is a direct summand of $s_{*} \mathcal{L}_{\Sigma}$ as sheaves of $\mathcal{A}_{\Delta}$-modules; in particular, $I H(\Sigma)$ contains $I H(\Delta)$ as a direct summand.

If $\Delta$ is an arbitrary fan, there exists a subdivision $s: \Sigma \rightarrow \Delta$ such that $\Sigma$ is simplicial, for example, the barycentric subdivision constructed below. Since $\mathcal{L}_{\Sigma} \simeq \mathcal{A}_{\Sigma}$, using the decomposition theorem we can consider sections of $\mathcal{L}_{\Delta}$ as conewise polynomial functions on the subdivision $\Sigma$.

Now let us construct a simplicial subdivision of an arbitrary fan $\Sigma$. First, suppose $\sigma \in \Sigma$ is a cone of dimension at least 2 such that any other cone $\tau \in \Sigma$ containing $\sigma$ can be written as a direct sum $\tau=\sigma \oplus \rho$ for some $\rho \in \operatorname{Link}_{\Sigma}(\sigma)$. If $\mathbb{R}_{\geq 0} v$ is a ray intersecting the relative interior of $\sigma$, we define the star subdivision of $\Sigma$ at $\mathbb{R}_{\geq 0} v$ to be the fan

$$
\left(\Sigma-\operatorname{Star}_{\Sigma}(\sigma)\right) \cup\left\{\mathbb{R}_{\geq 0} v+\tau+\rho \mid \tau \in \partial \sigma, \rho \in \operatorname{Link}_{\Sigma}(\sigma)\right\} .
$$

To simplify notation, we assume that the star subdivision of $\Sigma$ at $\mathbb{R}_{\geq 0} v$ where $v$ lies in a cone of dimension less than 2 does not change the fan.

Given an arbitrary fan $\Sigma$, for every cone $\sigma \in \Sigma$ let $v_{\sigma}$ be a vector in the relative interior of $\sigma$. We can perform a sequence of star subdivisions at $\mathbb{R}_{\geq 0} v_{\sigma}$, starting with cones $\sigma$ of maximal dimension, then cones of one smaller dimension, and so on. The resulting subdivision is simplicial and it is called a barycentric subdivision of $\Sigma$.

1.5. Evaluation map in the top degree cohomology. Following M. Brion [3] we construct an isomorphism $I H^{2 n}(\Sigma) \rightarrow \mathbb{R}$ for a complete $n$-dimensional fan $\Sigma$.

First assume that $\Sigma$ is a simplicial fan. For every $n$-dimensional cone $\sigma \in \Sigma$ we choose linear functions $H_{\sigma, 1}, \ldots, H_{\sigma, n}$ defining the facets of $\sigma$, such that $H_{\sigma, i}$ are non-negative on $\sigma$ and their wedge product has length 1 with respect to some metric on $\Lambda^{n} V^{*}$. Let $\Phi_{\sigma}$ be the product of the $H_{\sigma, i}$. Thus, up to a constant factor, $\Phi_{\sigma}$ is the unique degree $n$ polynomial that vanishes on $\partial \sigma$; the constant is determined by the metric on $\Lambda^{n} V^{*}$ together with the requirement that $\Phi_{\sigma}$ is non-negative on $\sigma$.

We identify $\mathcal{L}_{\Sigma}=\mathcal{A}_{\Sigma}$ and consider a section $f \in \mathcal{L}(\Sigma)$ as a conewise polynomial function. Then for a maximal cone $\sigma$, the stalk $f_{\sigma} \in \mathcal{A}_{\sigma}=A$ is a 
polynomial and we can define a rational function

$$
\langle f\rangle=\sum_{\sigma \in \Sigma, \operatorname{dim}} \frac{f_{\sigma=n}}{\Phi_{\sigma}} .
$$

Brion proved that the poles of this rational function cancel out so that the result is in fact a polynomial, thus defining a degree $-2 n$ map of $A$-modules

$$
\langle\cdot\rangle: \mathcal{L}(\Sigma) \longrightarrow A \text {. }
$$

Since $\langle\cdot\rangle$ maps $\mathcal{L}(\Sigma)^{2 n-2}$ to zero, it induces a map

$$
\langle\cdot\rangle: I H^{2 n}(\Sigma) \longrightarrow A^{0}=\mathbb{R} .
$$

Because this map is surjective (by considering a function $f$ supported on $\sigma$ such that $\left.f\right|_{\sigma}=\Phi_{\sigma}$ ) and $\operatorname{dim} I H^{2 n}(\Sigma)=\operatorname{dim} I H^{0}(\Sigma)=1$ (see the next section about Poincaré duality), it follows that the map $\langle\cdot\rangle$ is an isomorphism.

Now suppose that $\Delta$ is an arbitrary complete fan. We choose a simplicial subdivision $s: \Sigma \rightarrow \Delta$ and, using the decomposition theorem, embed

$$
\mathcal{L}_{\Delta} \subset s_{*} \mathcal{L}_{\Sigma}
$$

Restricting the map $\langle\cdot\rangle$ defined on the global sections of $\mathcal{L}_{\Sigma}$ to the global sections of $\mathcal{L}_{\Delta}$ gives us a map in cohomology

$$
\langle\cdot\rangle: I H^{2 n}(\Delta) \hookrightarrow I H^{2 n}(\Sigma) \longrightarrow \mathbb{R} .
$$

Since both top degree cohomology spaces have dimension 1, this composition is an isomorphism.

We sometimes use a subscript to indicate the fan for which the evaluation map is constructed:

$$
\langle\cdot\rangle_{\Delta}: I H^{2 n}(\Sigma) \longrightarrow \mathbb{R}
$$

1.6. Fans with boundary and the Poincaré pairing. As in [1] we consider a (possibly noncomplete) fan $\Sigma$ such that all maximal cones of $\Sigma$ have dimension $n$. Then the boundary $\partial \Sigma$ of $\Sigma$ consists of all faces of those $n-1$ dimensional cones in $\Sigma$ that are contained in only one $n$ dimensional cone. We further restrict ourselves to the case of quasi-convex fans [1] where the boundary $\partial \Sigma$ is a real homology manifold. The examples we wish to consider are complete fans (with $\partial \Sigma=\emptyset$ ), and fans of the type $\Sigma=\overline{\operatorname{Star}}_{\Delta}(\delta)$ for some cone $\delta$ in a complete fan $\Delta$.

If $\Sigma$ is a quasi-convex fan with boundary $\partial \Sigma$, let $\mathcal{L}(\Sigma, \partial \Sigma) \subset \mathcal{L}(\Sigma)$ be the space of sections vanishing on the boundary, and let

$$
I H(\Sigma, \partial \Sigma)=\overline{\mathcal{L}(\Sigma, \partial \Sigma)}
$$

(Note: in [1] the notation for $I H(\Sigma)$ and $I H(\Sigma, \partial \Sigma)$ is reversed compared to the one used here.)

It is proved in [囬] that for a quasi-convex fan $\Sigma$ both $\mathcal{L}(\Sigma)$ and $\mathcal{L}(\Sigma, \partial \Sigma)$ are finitely generated free $A$-modules. Moreover, there exists a bilinear nondegenerate map, called Poincaré pairing,

$$
I H^{n-k}(\Sigma) \times I H^{n+k}(\Sigma, \partial \Sigma) \longrightarrow \mathbb{R}, \quad k \in \mathbb{Z} .
$$

The pairing is constructed by considering sections of $\mathcal{L}_{\Sigma}$ as conewise polynomial functions on a simplicial subdivision $\Delta$ of $\Sigma$, multiplying these functions, and 
composing with the evaluation map $\langle\cdot\rangle: I H^{2 n}(\Delta) \longrightarrow \mathbb{R}$. We denote this pairing by

$$
(h, g) \longmapsto\langle h \cdot g\rangle .
$$

It is clear that the Poincaré pairing depends on the choice of a subdivision $\Delta$ and the embedding $\mathcal{L}(\Sigma) \subset \mathcal{L}(\Delta)$. We will fix one set of these choices in Section 4 and call the corresponding pairing the Poincaré pairing.

Another construction we will use later is the following. Let $\Sigma$ be a complete fan and $\Sigma_{1}, \Sigma_{2}$ subfans of $\Sigma$ such that

$$
\Sigma_{1} \cup \Sigma_{2}=\Sigma, \quad \Sigma_{1} \cap \Sigma_{2}=\partial \Sigma_{1}=\partial \Sigma_{2} .
$$

Assume that $\Sigma_{1}$, hence also $\Sigma_{2}$, is quasi-convex. Then we have an exact sequence of free $A$ modules

$$
0 \longrightarrow \mathcal{L}\left(\Sigma_{2}, \partial \Sigma_{2}\right) \longrightarrow \mathcal{L}(\Sigma) \longrightarrow \mathcal{L}\left(\Sigma_{1}\right) \longrightarrow 0,
$$

which splits non-canonically. Taking quotients by the ideal $I$ we get an exact sequence of cohomology spaces

$$
0 \longrightarrow I H\left(\Sigma_{2}, \partial \Sigma_{2}\right) \longrightarrow I H(\Sigma) \longrightarrow I H\left(\Sigma_{1}\right) \longrightarrow 0 .
$$

A splitting of the first sequence gives us a splitting of the second one.

\section{Projective Fans}

Let $\Sigma$ be a complete $n$-dimensional fan and $l \in \mathcal{A}^{2}(\Sigma)$ a conewise linear function on $\Sigma$. For an $n$-dimensional cone $\sigma \in \Sigma$ we consider $l_{\sigma}=\left.l\right|_{\sigma}$ as an element in $A^{2}$.

Definition 2.1. A function $l \in \mathcal{A}^{2}(\Sigma)$ is called strictly convex if $l_{\sigma}(v)<l(v)$ for any maximal cone $\sigma \in \Sigma$ and any $v \notin \sigma$. A complete fan $\Sigma$ is called projective if there exists a strictly convex function $l \in \mathcal{A}^{2}(\Sigma)$.

We note that if $f \in A$ is any linear function then $l$ is strictly convex if and only if $l+f$ is strictly convex. If $l$ is strictly convex, then modifying $l$ by some $f \in A$ if necessary, we may assume that $l(v)>0$ for $v \neq 0$. Then the set $\{v \mid l(v) \leq 1\}$ is a convex $n$-dimensional polytope, with faces corresponding to cones in $\Sigma$.

The main goal of this paper is to prove that if $l \in \mathcal{A}^{2}(\Sigma)$ is a strictly convex function on a complete fan then multiplication with $l$ induces an isomorphism

$$
l^{k}: I H^{n-k}(\Sigma) \longrightarrow I H^{n+k}(\Sigma) .
$$

Definition 2.2. Let $H$ be a finite dimensional graded vector space and $l$ : $H \rightarrow H$ a degree 2 linear map. We say that $l$ is a Lefschetz operation centered at degree $n$ if

$$
l^{k}: H^{n-k} \longrightarrow H^{n+k}
$$

is an isomorphism for all $k>0$.

If $l: H \rightarrow H$ is a Lefschetz operation centered at degree $n$, we define the primitive part of $H$ with respect to $l$ :

$$
P_{l}^{n-k}=H^{n-k} \cap \operatorname{ker} l^{k+1} .
$$

Then if $\left\{p_{i}\right\}_{i=1}^{N}$ is a homogeneous basis of $P_{l}$, one can construct a basis of $H$ of the form $\left\{l^{j_{i}} p_{i} \mid 1 \leq i \leq N, 0 \leq j_{i} \leq n-\operatorname{deg}\left(p_{i}\right)\right\}$. 
2.1. Hodge-Riemann-Minkowski bilinear relations. Let $\Sigma$ be a complete $n$-dimensional fan and $l \in \mathcal{A}^{2}(\Sigma)$ a strictly convex function on $\Sigma$. Using the Poincaré pairing we define a quadratic form $Q_{l}$ on $I H^{n-k}(\Sigma)$ :

$$
Q_{l}(h)=\left\langle l^{k} h \cdot h\right\rangle .
$$

Definition 2.3. We say that $Q_{l}$ satisfies Hodge-Riemann-Minkowski bilinear relations if for any $k \geq 0$ the quadratic form

$$
(-1)^{\frac{n-k}{2}} Q_{l}
$$

is positive definite when restricted to the primitive cohomology

$$
I P_{l}^{n-k}(\Sigma)=I H^{n-k}(\Sigma) \cap \operatorname{ker} l^{k+1} .
$$

It is clear that if $Q_{l}$ satisfies the Hodge-Riemann-Minkowski bilinear relations then $l^{k}: I H^{n-k}(\Sigma) \rightarrow I H^{n+k}(\Sigma)$ is injective for all $k>0$. Poincaré duality then implies that these maps are isomorphisms and $l$ defines a Lefschetz operation on $I H(\Sigma)$ centered at degree $n$.

It is also useful to write the Hodge-Riemann-Minkowski bilinear relations in terms of the signature of $Q_{l}$.

Lemma 2.4. $Q_{l}$ satisfies Hodge-Riemann-Minkowski bilinear relations if and only if for any $k \leq n$ the signature of the quadratic form $Q_{l}$ on $I H^{k}(\Sigma)$ is

$$
\left(\sum_{0 \leq j \leq k, 4 \mid j} h^{j}-h^{j-2}, \sum_{0 \leq j \leq k, 4 \nmid j} h^{j}-h^{j-2}\right),
$$

where $h^{j}=\operatorname{dim} I H^{j}(\Sigma)$.

Proof. Note that each of the two statements whose equivalence we are proving implies that $Q_{l}$ is nondegenerate on $I H^{k}(\Sigma)$. Hence we may assume that $l$ defines a Lefschetz operation and, in particular, that $l: I H^{k-2}(\Sigma) \rightarrow$ $I H^{k}(\Sigma)$ is injective for $k \leq n$. Thus we have a decomposition

$$
I H^{k}(\Sigma)=l \cdot I H^{k-2}(\Sigma) \oplus I P_{l}^{k}(\Sigma) .
$$

Since both the Poincaré pairing and the operation $l$ are defined by multiplication of functions, it follows that $l$ is self-adjoint with respect to the pairing:

$$
\langle l x \cdot y\rangle=\langle x \cdot l y\rangle .
$$

This implies that for $k \leq n$ we have

(a) $Q_{l}(l x)=Q_{l}(x)$ for any $x \in I H^{k-2}(\Sigma)$.

(b) The primitive cohomology $I P_{l}^{k}(\Sigma)$ is orthogonal to $l \cdot I H^{k-2}(\Sigma)$ with respect to the bilinear form $B_{l}$ on $I H^{k}(\Sigma)$ :

$$
B_{l}(x, y)=\left\langle l^{k} x \cdot y\right\rangle \text {. }
$$

Thus the decomposition $I H^{k}(\Sigma)=l \cdot I H^{k-2}(\Sigma) \oplus I P_{l}^{k}(\Sigma)$ is orthogonal with respect to the bilinear form $B_{l}$ and the signature of $Q_{l}$ on $I H^{k}(\Sigma)$ is the sum of the signatures of $Q_{l}$ on $I H^{k-2}(\Sigma)$ and on $I P_{l}^{k}(\Sigma)$. The claim of the lemma now follows by induction on $k$.

McMullen in [7] proved that the Hodge-Riemann-Minkowski relations hold if the fan is simplicial (or equivalently, the corresponding polytope is simple). His proof, however, was written in terms of the polytope algebra and the weight spaces of a polytope. In the following, we want to assume that the 
Hodge-Riemann-Minkowski relations hold on the intersection cohomology of a simplicial fan when the quadratic form is defined by multiplication of functions as above. There are two ways we can make this assumption valid. First, if the fan is rational, then the algebra $\mathcal{A}(\Sigma)$ is isomorphic to the equivariant cohomology $H_{T}\left(X_{\Sigma}\right)$ of the corresponding toric variety (e.g., see [5]). Then the Hodge-Riemann-Minkowski relations follow from the corresponding relations on $H_{T}\left(X_{\Sigma}\right)$. The case of a nonrational simplicial fan follows by a small deformation to a rational fan, as in the original proof of Stanley [B]. Second, if we wish to stay in the realm of algebra and combinatorics, we can apply the Koszul functor [5] to the cellular complex [1, 2] computing the global sections $\mathcal{A}(\Sigma)$. As a result we recover the weight spaces together with the product rule used by McMullen. Since this calculation is straight-forward, we leave it to the reader.

\section{Products OF FAnS}

3.1. The Künneth theorem. We consider fans $\Sigma$ in $V$ and $\Delta$ in $W$, and their product fan $\Sigma \times \Delta$ in $V \times W$. Let $\pi_{1}: V \times W \rightarrow V$ and $\pi_{2}: V \times W \rightarrow W$ be the two projections, inducing the maps of fans $\pi_{1}: \Sigma \times \Delta \rightarrow \Sigma$ and $\pi_{2}: \Sigma \times \Delta \rightarrow \Delta$. Then it is easy to see that

$$
\pi_{1}^{-1} \mathcal{A}_{\Sigma} \otimes \pi_{2}^{-1} \mathcal{A}_{\Delta} \simeq \mathcal{A}_{\Sigma \times \Delta}
$$

Indeed, there is a natural morphism $\pi_{1}^{-1} \mathcal{A}_{\Sigma} \otimes \pi_{2}^{-1} \mathcal{A}_{\Delta} \rightarrow \mathcal{A}_{\Sigma \times \Delta}$ defined by pullback of functions. On each cone $\sigma \times \delta \in \Sigma \times \Delta$ this map is an isomorphism $\mathcal{A}_{\sigma} \otimes$ $\mathcal{A}_{\delta} \stackrel{\sim}{\rightarrow} \mathcal{A}_{\sigma \times \delta}$, hence it is an isomorphism of sheaves. Using this isomorphism, we can view $\pi_{1}^{-1} \mathcal{L}_{\Sigma} \otimes \pi_{2}^{-1} \mathcal{L}_{\Delta}$ as a sheaf of $\mathcal{A}_{\Sigma \times \Delta}$ modules.

Theorem 3.1. Let $\Sigma$ be a fan in $V$ and $\Delta$ a fan in $W$. Then

$$
\pi_{1}^{-1} \mathcal{L}_{\Sigma} \otimes \pi_{2}^{-1} \mathcal{L}_{\Delta} \simeq \mathcal{L}_{\Sigma \times \Delta}
$$

Moreover, there exists an isomorphism of $\operatorname{Sym}(V \times W)^{*} \simeq \operatorname{Sym} V^{*} \otimes \operatorname{Sym} W^{*}$ modules

$$
\mathcal{L}(\Sigma) \otimes \mathcal{L}(\Delta) \simeq \mathcal{L}(\Sigma \times \Delta)
$$

Proof. We prove both statements by induction on the dimension of the fan $\Sigma \times \Delta$, the case where either $\Sigma$ or $\Delta$ has dimension 0 being trivial. Let us denote $A=\operatorname{Sym} V^{*}$ and $B=\operatorname{Sym} W^{*}$.

We start by proving the second statement, assuming the first. We have

$$
\left(\pi_{1}\right)_{*} \mathcal{L}_{\Sigma \times \Delta} \simeq\left(\pi_{1}\right)_{*}\left(\pi_{1}^{-1} \mathcal{L}_{\Sigma} \otimes \pi_{2}^{-1} \mathcal{L}_{\Delta}\right) \simeq \mathcal{L}_{\Sigma} \otimes \mathcal{L}(\Delta)
$$

Taking global sections of this push-forward gives the second statement.

Next we prove the first statement of the theorem, assuming the theorem for fans of smaller dimension. We have to show that the sheaf $\mathcal{M}=\pi_{1}^{-1} \mathcal{L}_{\Sigma} \otimes$ $\pi_{2}^{-1} \mathcal{L}_{\Delta}$ satisfies the three conditions in the definition of an equivariant intersection cohomology sheaf. Note that for any cone $\sigma \times \delta \in \Sigma \times \Delta$ we have

$$
\mathcal{M}_{\sigma \times \delta}=\mathcal{L}_{\sigma} \otimes \mathcal{L}_{\delta}
$$

From this we deduce the normalization condition $\mathcal{M}_{0 \times 0}=\mathbb{R} \otimes \mathbb{R}=\mathbb{R}$ and the local freeness of $\mathcal{M}$ : since $\mathcal{L}_{\sigma}, \mathcal{L}_{\delta}$ are free $\mathcal{A}_{\sigma}, \mathcal{A}_{\delta}$ modules, respectively, it follows that $\mathcal{L}_{\sigma} \otimes \mathcal{L}_{\delta}$ is a free $\mathcal{A}_{\sigma} \otimes \mathcal{A}_{\delta}$ module. 
It remains to show that the restriction map $\mathcal{M}_{\sigma \times \delta} \rightarrow \mathcal{M}(\partial(\sigma \times \delta))$ induces an isomorphism after taking quotients by the ideal $I$. From the formula

$$
\partial(\sigma \times \delta)=\partial \sigma \times \delta \bigcup_{\partial \sigma \times \partial \delta} \sigma \times \partial \delta
$$

we get that $\mathcal{M}(\partial(\sigma \times \delta))$ is the kernel of the map

$$
\mathcal{M}(\partial \sigma \times \delta) \oplus \mathcal{M}(\sigma \times \partial \delta) \stackrel{\left(\begin{array}{c}
\text { res } \\
- \text { res }
\end{array}\right)}{\longrightarrow} \mathcal{M}(\partial \sigma \times \partial \delta),
$$

defined by restrictions with appropriate signs. Also note that the restriction map $\mathcal{M}_{\sigma \times \delta} \rightarrow \mathcal{M}(\partial(\sigma \times \delta))$ is defined by

$$
\mathcal{M}_{\sigma \times \delta} \stackrel{\left(\begin{array}{c}
\text { res } \\
\text { res }
\end{array}\right)}{\longrightarrow} \mathcal{M}(\partial \sigma \times \delta) \oplus \mathcal{M}(\sigma \times \partial \delta) .
$$

We now apply induction assumption to the fans $\partial \sigma \times \delta, \sigma \times \partial \delta$ and $\partial \sigma \times \partial \delta$ to get an exact sequence

$$
0 \longrightarrow \mathcal{M}(\partial(\sigma \times \delta)) \longrightarrow \mathcal{L}(\partial \sigma) \otimes \mathcal{L}_{\delta} \oplus \mathcal{L}_{\sigma} \otimes \mathcal{L}(\partial \delta) \longrightarrow \mathcal{L}(\partial \sigma) \otimes \mathcal{L}(\partial \delta) \longrightarrow 0
$$

where exactness on the right follows from the flabbiness of $\mathcal{L}$. The restriction map from $\mathcal{M}_{\sigma \times \delta}=\mathcal{L}_{\sigma} \otimes \mathcal{L}_{\delta}$ to $\mathcal{M}(\partial(\sigma \times \delta))$ is defined by a map to the middle term of this sequence. Assuming for a moment that this sequence remains exact after tensoring with the $A \otimes B$-module $\mathbb{R}$, and using that the restriction maps induce isomorphisms $\overline{\mathcal{L}}_{\sigma} \simeq \overline{\mathcal{L}(\partial \sigma)}$ and $\overline{\mathcal{L}}_{\delta} \simeq \overline{\mathcal{L}(\partial \delta)}$, we get an exact sequence

$0 \rightarrow \overline{\mathcal{M}(\partial(\sigma \times \delta))} \longrightarrow \overline{\mathcal{L}(\partial \sigma)} \otimes \overline{\mathcal{L}(\partial \delta)} \oplus \overline{\mathcal{L}(\partial \sigma)} \otimes \overline{\mathcal{L}(\partial \delta)} \stackrel{\left({ }_{\text {IId }}\right.}{\longrightarrow} \overline{\mathcal{L}(\partial \sigma)} \otimes \overline{\mathcal{L}(\partial \delta)} \rightarrow 0$.

From this we get

$$
\overline{\mathcal{M}(\partial(\sigma \times \delta))} \simeq \overline{\mathcal{L}(\partial \sigma)} \otimes \overline{\mathcal{L}(\partial \delta)} \simeq \overline{\mathcal{L}}_{\sigma} \otimes \overline{\mathcal{L}}_{\delta}
$$

and the restriction map from $\overline{\mathcal{M}}_{\sigma \times \delta} \simeq \overline{\mathcal{L}}_{\sigma} \otimes \overline{\mathcal{L}}_{\delta}$ to it is the identity.

Now let us prove that tensoring with $\mathbb{R}$ keeps the sequence above exact. Following a similar argument in [1], we show that the map

$$
\operatorname{Tor}_{1}^{A \otimes B}\left(\mathcal{L}(\partial \sigma) \otimes \mathcal{L}_{\delta} \oplus \mathcal{L}_{\sigma} \otimes \mathcal{L}(\partial \delta), \mathbb{R}\right) \longrightarrow \operatorname{Tor}_{1}^{A \otimes B}(\mathcal{L}(\partial \sigma) \otimes \mathcal{L}(\partial \delta), \mathbb{R})
$$

is an isomorphism. For any cone $\tau$, let $\mathcal{K}_{\tau}=\mathcal{L}([\tau], \partial \tau)$ be the kernel of the restriction map $\mathcal{L}_{\tau} \rightarrow \mathcal{L}(\partial \tau)$. Since $[\tau]$ is quasi-convex in its span, $\mathcal{K}_{\tau}$ is a free $\mathcal{A}_{\tau}$-module. Hence the sequence

$$
0 \longrightarrow \mathcal{K}_{\tau} \longrightarrow \mathcal{L}_{\tau}
$$

is a free resolution of the $\mathcal{A}_{\tau}$-module $\mathcal{L}(\partial \tau)$. Also, the induced map $\overline{\mathcal{K}}_{\tau} \rightarrow \overline{\mathcal{L}}_{\tau}$ is zero because $\overline{\mathcal{L}}_{\tau} \simeq \overline{\mathcal{L}(\partial \tau)}$. Using such resolutions, the map of the $\operatorname{Tor}_{1}^{A \otimes B}$ can be expressed as the isomorphism

$$
\overline{\mathcal{K}}_{\sigma} \otimes \overline{\mathcal{L}}_{\delta} \oplus \overline{\mathcal{L}}_{\sigma} \otimes \overline{\mathcal{K}}_{\delta} \stackrel{\left({ }^{\text {Id }}{ }_{\text {Id }}\right)}{\longrightarrow} \overline{\mathcal{K}}_{\sigma} \otimes \overline{\mathcal{L}}_{\delta} \oplus \overline{\mathcal{L}}_{\sigma} \otimes \overline{\mathcal{K}}_{\delta}
$$

Corollary 3.2. Let $\Sigma$ be a quasi-convex fan in $V$ and $\Delta$ a quasi-convex fan in $W$. Then $\Sigma \times \Delta$ is also quasi-convex and there exists an isomorphism of $\operatorname{Sym}(V \times W)^{*} \simeq \operatorname{Sym} V^{*} \otimes \operatorname{Sym} W^{*}$ modules

$$
\mathcal{L}(\Sigma, \partial \Sigma) \otimes \mathcal{L}(\Delta, \partial \Delta) \simeq \mathcal{L}(\Sigma \times \Delta, \partial(\Sigma \times \Delta)) .
$$


Proof. Theorem 3.8 in 11] shows that a fan $\Theta$ is quasi-convex if and only if the module $\mathcal{L}(\Theta)$ is free. Since $\mathcal{L}(\Sigma \times \Delta) \simeq \mathcal{L}(\Sigma) \otimes \mathcal{L}(\Delta)$ is free, the product fan is quasi-convex.

Again by [1] a quasi-convex $n$-dimensional fan $\Theta$ satisfies the Poincaré duality

$$
\operatorname{dim} I H^{n-k}(\Theta)=\operatorname{dim} I H^{n+k}(\Theta, \partial \Theta), \quad k \in \mathbb{Z} .
$$

Applying this to each of the three spaces in the inclusion

$$
\mathcal{L}(\Sigma, \partial \Sigma) \otimes \mathcal{L}(\Delta, \partial \Delta) \hookrightarrow \mathcal{L}(\Sigma \times \Delta, \partial(\Sigma \times \Delta)),
$$

we get that both sides are free modules of the same rank (in each degree). Thus the inclusion map must be an isomorphism.

Let us assume further that $\Sigma$ and $\Delta$ are complete projective fans with strictly convex conewise linear functions $l_{\Sigma}$ and $l_{\Delta}$ on them. Then the function $l=$ $\pi_{1}^{*} l_{\Sigma}+\pi_{2}^{*} l_{\Delta}$ is strictly convex on the product fan $\Sigma \times \Delta$. We wish to state that if $Q_{l_{\Sigma}}$ and $Q_{l_{\Delta}}$ both satisfy the Hodge-Riemann-Minkowski bilinear relations on the respective cohomology spaces, then so does $Q_{l}$. For this we need to assume that the representation of sections $\mathcal{L}(\Sigma \times \Delta) \simeq \mathcal{L}(\Sigma) \otimes \mathcal{L}(\Delta)$ as conewise polynomial functions on some subdivision is induced by the representation of sections $\mathcal{L}(\Sigma)$ and $\mathcal{L}(\Delta)$ as functions, and that the evaluation map satisfies:

$$
\left\langle h_{1} \otimes h_{2}\right\rangle_{\Sigma \times \Delta}=\left\langle h_{1}\right\rangle_{\Sigma} \cdot\left\langle h_{2}\right\rangle_{\Delta}, \quad h_{1} \in I H(\Sigma), h_{2} \in I H(\Delta) .
$$

If these conditions are satisfied then we have:

Corollary 3.3. If $Q_{l_{\Sigma}}$ and $Q_{l_{\Delta}}$ both satisfy the Hodge-Riemann-Minkowski bilinear relations then so does $Q_{l}$.

Proof. We choose a homogeneous basis $\left\{p_{i}\right\}$ of $\operatorname{IP}(\Sigma)$, orthogonal with respect to the bilinear form $B_{l_{\Sigma}}(x, y)=\left\langle l_{\Sigma}^{k} x \cdot y\right\rangle$, and a similar orthogonal basis $\left\{q_{j}\right\}$ of $I P(\Delta)$. Then it suffice to show that $Q_{l}$ satisfies the HodgeRiemann-Minkowski relations on the subspace $S \subset I H(\Sigma \times \Delta)$ with basis

$$
\left\{l_{\Sigma}^{\alpha} p \otimes l_{\Delta}^{\beta} q\right\}_{0 \leq \alpha \leq k_{1}, 0 \leq \beta \leq k_{2}}
$$

generated by one $p=p_{i_{0}}$ and one $q=q_{j_{0}}$. Since $l$ acts by multiplication with $l_{\Sigma} \otimes 1+1 \otimes l_{\Delta}$, it is clear that the signature of $Q_{l}$ on $S$ only depends on the numbers $k_{1}, k_{2}$ and the signs of $Q_{l_{\Sigma}}(p), Q_{l_{\Delta}}(q)$.

One can give a geometric proof that $Q_{l}$ satisfies the Hodge-Riemann-Minkowski relations on $S$ by comparing it with the quadratic form $Q_{A}$ defined on the singular cohomology $H\left(\mathbb{C P}^{k_{1}} \times \mathbb{C P}^{k_{2}}, \mathbb{C}\right)$, where the Lefschetz action is given by intersection with an ample divisor $A$, and the pairing is the usual cup product. Then $Q_{A}$ satisfies the classical Hodge-Riemann bilinear relations [6]. The combinatorial analog of this is to construct two simplicial fans $\Pi^{k_{1}}$ and $\Pi^{k_{2}}$ corresponding to the toric varieties $\mathbb{C P}^{k_{1}}$ and $\mathbb{C P}^{k_{2}}$, together with strictly convex functions on them. Then the Hodge-Riemann-Minkowski relations [7] for the simplicial fan $\Pi^{k_{1}} \times \Pi^{k_{2}}$ imply the the same relations for $Q_{l}$ above.

3.2. Skew products. Consider two fans $\Sigma$ in $V$ and $\Delta$ in $W$. Let $\phi:|\Sigma| \rightarrow W$ be a map which is linear on each cone $\sigma \in \Sigma$, and let $\Gamma_{\sigma}(\phi)$ be the graph of $\phi$ restricted to $\sigma$. We define the fan $\Sigma \times_{\phi} \Delta$ in $V \times W$ :

$$
\Sigma \times_{\phi} \Delta=\left\{\Gamma_{\sigma}(\phi)+\delta \mid \sigma \in \Sigma, \delta \in \Delta\right\} .
$$


Then if $\phi$ is the zero map, we recover the usual product. For a general $\phi$ we only get a conewise linear isomorphism

$$
\Phi: \Sigma \times \Delta \longrightarrow \Sigma \times_{\phi} \Delta
$$

defined by $|\Phi|(v, w)=(v, w+\phi(v))$ for $v \in|\Sigma|$ and $w \in|\Delta|$.

Since $\Phi$ is a conewise linear isomorphism of fans, it induces an isomorphism of vector spaces

$$
\mathcal{L}\left(\Sigma \times_{\phi} \Delta\right) \simeq \mathcal{L}(\Sigma \times \Delta) \simeq \mathcal{L}(\Sigma) \otimes \mathcal{L}(\Delta)
$$

Let $A$ and $B$ be the rings of polynomial functions on $V$ and $W$, respectively. Then the isomorphism of global sections is in fact an isomorphism of $A$-modules. A functions $f \in B$ acts on the tensor product by multiplication with $\Phi^{*}(1 \otimes f)$. In particular, if $f \in B$ is linear, then

$$
\Phi^{*}(1 \otimes f)=1 \otimes f+f \circ \phi \otimes 1 .
$$

3.3. Local product structures. We say that a fan $\Sigma$ in $V$ has a local product structure at a cone $\sigma \in \Sigma$ if

$$
\overline{\operatorname{Star}}_{\Sigma}(\sigma) \simeq \Lambda \times_{\phi}[\sigma]
$$

for some fan $\Lambda$ in $W$, where $V=W \oplus \operatorname{Span}(\sigma)$, and some function $\phi:|\Lambda| \rightarrow$ $\operatorname{Span}(\sigma)$. Equivalently, $\Sigma$ has a local product structure at $\sigma$ if every cone $\tau \in \Sigma$ containing $\sigma$ can be written as a direct sum $\tau=\rho \oplus \sigma$ for some $\rho \in \operatorname{Link}_{\Sigma}(\sigma)$. If $\pi: \overline{\operatorname{Star}}_{\Sigma}(\sigma) \rightarrow \Lambda$ is the projection from $\operatorname{Span}(\sigma)$ then $\pi$ restricts to a conewise linear isomorphism $\operatorname{Link}_{\Sigma}(\sigma) \rightarrow \Lambda$.

As an example, when we perform a star subdivision of the fan $\Sigma$ at $\mathbb{R}_{\geq 0} v$, where $v$ lies in the relative interior of a cone $\sigma$, we require $\Sigma$ to have a local product structure at $\sigma$. The subdivision $\hat{\Sigma}$ then also has a local product structure at $\mathbb{R}_{\geq 0} v \in \hat{\Sigma}$.

Lemma 3.4. Assume that $\Sigma$ has a local product structure at a simplicial cone $\sigma \in \Sigma$. Let $\Delta=\overline{\operatorname{Star}}_{\Sigma}(\sigma)$; then with notation as above,

$$
\pi^{*} \mathcal{L}_{\Lambda} \simeq \mathcal{L}_{\Delta}
$$

Moreover, $\pi^{*}$ induces an isomorphism of A-modules

$$
\mathcal{L}(\Delta) \simeq \mathcal{L}(\Lambda) \otimes_{B} A
$$

hence also an isomorphism $\operatorname{IH}(\Delta) \simeq I H(\Lambda)$.

Proof. A cone $\tau \in \Delta$ has the form $\tau=\Gamma_{\rho}(\phi) \oplus \sigma^{\prime}$ for some $\rho \in \Lambda$ and $\sigma^{\prime}$ a face of $\sigma$. Since $\sigma^{\prime}$ is simplicial, $\mathcal{L}_{\sigma^{\prime}} \simeq \mathcal{A}_{\sigma^{\prime}}$ and we have an isomorphism of $\mathcal{A}_{\tau}$ modules

$$
\mathcal{L}_{\tau} \simeq \mathcal{L}_{\Gamma_{\rho}(\phi)} \otimes \mathcal{A}_{\sigma^{\prime}} \simeq \mathcal{L}_{\rho} \otimes_{\mathcal{A}_{\rho}} \mathcal{A}_{\tau}
$$

This proves the first isomorphism. The isomorphism above also induces an isomorphism of $A$-modules

$$
\mathcal{L}_{\tau} \simeq \mathcal{L}_{\rho} \otimes_{B} A
$$

Let $M \subset \Lambda$ be the set of maximal cones, i.e., cones which are not proper faces of any other cones. Then the cones $\Gamma_{\rho}(\phi) \oplus \sigma$ for $\rho \in M$ are the maximal 
cones of $\Delta$. Using the covering by maximal cones, $\mathcal{L}(\Delta)$ is the kernel of the restriction map

$$
\bigoplus_{\rho \in M} \mathcal{L}_{\Gamma_{\rho}(\phi) \oplus \sigma} \longrightarrow \bigoplus_{\rho_{1}, \rho_{2} \in M} \mathcal{L}_{\Gamma_{\rho_{1}}(\phi) \oplus \sigma \cap \Gamma_{\rho_{2}}(\phi) \oplus \sigma}
$$

We can write this sequence of $A$-modules as

$$
\left(\bigoplus_{\rho \in M} \mathcal{L}_{\rho} \longrightarrow \bigoplus_{\rho_{1}, \rho_{2} \in M} \mathcal{L}_{\rho_{1} \cap \rho_{2}}\right) \otimes_{B} A
$$

Since the kernel of the map in the parentheses is the $B$-module $\mathcal{L}(\Lambda)$, we get the stated isomorphism of global sections and intersection cohomologies.

\section{Distinguished EMBEDDings}

The Poincaré pairing, and hence the definition of the quadratic form $Q_{l}$, depend on the representation of sections of $\mathcal{L}_{\Sigma}$ as conewise polynomial functions on some subdivision of $\Sigma$. The goal of this section is to fix one such representation.

4.1. Primitive embeddings. By the definition of the sheaf $\mathcal{L}$, for any cone $\sigma$ we have

$$
\mathcal{L}_{\sigma} \simeq \overline{\mathcal{L}(\partial \sigma)} \otimes \mathcal{A}_{\sigma}
$$

We start by recalling how $\overline{\mathcal{L}(\partial \sigma)}$ can be identified with the primitive cohomology of the "flattened boundary fan" [1, 2].

By restricting to the span of $\sigma$ if necessary, we may assume that $\sigma$ is an $n$-dimensional cone in $V$. Let $v$ be a vector in the relative interior of $\sigma$, $W=V / \mathbb{R} v$, and let $\pi: V \rightarrow W$ be the projection. Then $\pi$ induces a conewise linear isomorphism

$$
\pi: \partial \sigma \rightarrow \Lambda
$$

for a complete fan $\Lambda$ in $\mathrm{W}$. If we let $B=\operatorname{Sym} W^{*}$ be the ring of polynomial functions on $W$ and $A=B[x]$ for some linear function $x$ not vanishing on $v$, then $\pi^{*}$ defines an isomorphism of $B$-modules

$$
\mathcal{L}(\partial \sigma) \simeq \mathcal{L}(\Lambda)
$$

Multiplication of sections $\mathcal{L}(\partial \sigma)$ with $x$ corresponds via this isomorphism to multiplication of sections $\mathcal{L}(\Lambda)$ with a strictly convex conewise linear function $\lambda$ on $\Lambda$, where

$$
\lambda=x \circ\left(\left.\pi\right|_{\partial \sigma}\right)^{-1} .
$$

Thus, if we assume the hard Lefschetz theorem for the lower dimensional fan $\Lambda$ (an assumption we will make everywhere in this section), we have

$$
\overline{\mathcal{L}(\partial \sigma)} \simeq \overline{\mathcal{L}(\Lambda)} / \lambda \cdot \overline{\mathcal{L}(\Lambda)} \simeq I P_{\lambda}(\Lambda) .
$$

We note that this construction does not depend on a particular choice of $x$; indeed, modulo the maximal ideal $I_{B} \subset B$ generated by linear functions, $x$ is determined up to a constant factor.

Now let $[\hat{\sigma}]$ be the star subdivision of $[\sigma]$ at $\mathbb{R}_{\geq 0} v$. Then by Lemma 3.4, the pullback $\pi^{*}$ induces an isomorphism

$$
\overline{\mathcal{L}([\hat{\sigma}])} \simeq \overline{\mathcal{L}(\Lambda)}=I H(\Lambda),
$$


hence multiplication with $\pi^{*}(\lambda)$ defines a Lefschetz operation on $\overline{\mathcal{L}([\hat{\sigma}])}$ centered at degree $\operatorname{dim} \sigma-1$.

Definition 4.1. Let $s: \hat{\Sigma} \rightarrow \Sigma$ be the star subdivision of a fan $\Sigma$ at $\mathbb{R}_{\geq 0} v$, where $v$ lies in the relative interior of a cone $\sigma \in \Sigma$. We say that a direct embedding (i.e, an embedding as a direct summand of locally free $\mathcal{A}_{\Sigma}$ modules)

$$
\mathcal{L}_{\Sigma} \subset s_{*} \mathcal{L}_{\hat{\Sigma}}
$$

is a primitive embedding if the induced embedding

$$
\overline{\mathcal{L}([\sigma])} \subset \overline{\mathcal{L}([\hat{\sigma}])}
$$

identifies $\overline{\mathcal{L}([\sigma])}$ with the $\lambda$-primitive part of $\overline{\mathcal{L}([\hat{\sigma}])}$. Here $[\hat{\sigma}] \subset \hat{\Sigma}$ is the star subdivision of $[\sigma]$ at $\mathbb{R}_{\geq 0} v$, and $\lambda$ is a conewise linear function on $[\hat{\sigma}]$ such that $\lambda(v)=0,\left.\lambda\right|_{\partial \sigma}=\left.x\right|_{\partial \sigma}$ for some linear function $x \in A, x(v) \neq 0$.

4.2. Distinguished subdivisions. We now specify certain distinguished simplicial subdivisions of a fan $\Sigma$ and distinguished representations of sections of $\mathcal{L}_{\Sigma}$ as conewise polynomial functions on the subdivision.

Definition 4.2. A distinguished subdivision of a fan $\Sigma$ is a sequence

$$
\Sigma_{0} \stackrel{s_{1}}{\longrightarrow} \Sigma_{1} \stackrel{s_{2}}{\longrightarrow} \ldots \stackrel{s_{N}}{\longrightarrow} \Sigma_{N}=\Sigma,
$$

where $s_{i}: \Sigma_{i-1} \rightarrow \Sigma_{i}$ is a star subdivision at $\mathbb{R}_{\geq 0} v_{i}$ for $i=1, \ldots, N$, satisfying the following conditions

(1) $\Sigma_{0}$ is simplicial.

(2) There exists a subfan $\Sigma^{s} \subset \Sigma$, the "singular" subfan, such that the sequence of subdivisions $s_{i}$ is induced by a barycentric subdivision of $\Sigma^{s}$. In other words, $v_{i} \in\left|\Sigma^{s}\right|$, and the sequence of star subdivisions of $\Sigma^{s}$ at $\mathbb{R}_{>0} v_{i}$ for $i=N, \ldots, 1$ is a barycentric subdivision of $\Sigma^{s}$.

(3) The subfan $\Sigma^{s}$ has the property that $\left|\Sigma^{s}\right| \cap \sigma$ is a face of $\sigma$ for any cone $\sigma \in \Sigma$.

The second condition of the definition implies that any cone $\tau \in \Sigma^{s}$ of dimension at least 2 contains a unique $v_{i}$ in its relative interior for some $1 \leq$ $i \leq N$. We denote this $v_{i}$ by $v_{\tau}$.

As an example, a barycentric subdivision is a distinguished subdivision of $\Sigma$, with $\Sigma^{s}=\Sigma$. Also, if $s=s_{N} \circ \ldots \circ s_{1}$ is a distinguished subdivision of $\Sigma=\Sigma_{N}$, then $t=s_{N-1} \circ \ldots \circ s_{1}$ is a distinguished subdivision of $\Sigma_{N-1}$, with $\Sigma_{N-1}^{s}=\Sigma^{s}-\left\{\sigma_{N}\right\}$, where $\sigma_{N} \in \Sigma$ is the cone containing $v_{N}$ in its relative interior.

Suppose we have a distinguished subdivision of $\Sigma$. Given a cone $\sigma \in \Sigma$, we can express $\sigma=\tau \oplus \rho$, where $\tau=\sigma \cap\left|\Sigma^{s}\right|$ and $\rho$ is a simplicial cone. Using the Künneth theorem and the definition of the sheaf $\mathcal{L}$, we have

$$
\mathcal{L}_{\sigma} \simeq \mathcal{L}_{\tau} \otimes \mathcal{L}_{\rho} \simeq \overline{\mathcal{L}(\partial \tau)} \otimes_{\mathcal{A}_{\tau}} \mathcal{A}_{\sigma}
$$

Thus, a set of $\mathcal{A}_{\tau}$ module generators of $\mathcal{L}(\partial \tau)$ can be chosen as the $\mathcal{A}_{\sigma}$ module generators of $\mathcal{L}_{\sigma}$. When representing sections of $\mathcal{L}$ as functions, we impose this condition on generators explicitly. 
Definition 4.3. Let $s=s_{N} \circ \ldots \circ s_{1}$ be a distinguished subdivision of a fan $\Sigma$. Given an embedding of $\mathcal{A}_{\Sigma}$ modules

$$
\mathcal{L}_{\Sigma} \subset s_{*} \mathcal{A}_{\Sigma_{0}}
$$

we have a representation of sections of $\mathcal{L}_{\Sigma}$ as conewise polynomial functions on $\Sigma_{0}$. We say that this representation is distinguished if it satisfies the following condition. For any cone $\sigma \in \Sigma$, write $\sigma=\tau \oplus \rho$, where $\tau=\sigma \cap\left|\Sigma^{s}\right|$ and $\rho$ is a simplicial cone. Then the condition is that there exist generators of the $\mathcal{A}_{\sigma}$ module $\mathcal{L}_{\sigma}$ represented by functions on $\partial \tau$, pulled back to $\sigma$ by the projection map

$$
\pi: \operatorname{Span} \sigma \longrightarrow \operatorname{Span} \sigma /\left(\mathbb{R} v_{\tau}+\operatorname{Span} \rho\right) .
$$

We remark that choosing a distinguished embedding $\mathcal{L}_{\Sigma} \subset s_{*} \mathcal{A}_{\Sigma_{0}}$ is equivalent to choosing such an embedding for the sheaves restricted to the subfan $\Sigma^{s}$.

Lemma 4.4. Consider a distinguished subdivision $s=s_{N} \circ \ldots \circ s_{1}$ of a fan $\Sigma$ and a distinguished representation of sections of $\mathcal{L}_{\Sigma}$ as functions on $\Sigma_{0}$. Let $\rho \in \Sigma$ be a cone such that $\rho \cap \Sigma^{s}=\{0\}$, and denote $\Delta=\overline{\operatorname{Star}}_{\Sigma} \rho$. Then $\Sigma$ has a local product structure at $\rho$ :

$$
\Delta=\Lambda \times_{\phi}[\rho],
$$

and there exist generators of the $A$ module $\mathcal{L}(\Delta)$ represented by functions on (the subdivision of) $\Lambda$, pulled back to $\Delta$ by the projection

$$
\pi: \Delta \longrightarrow \Lambda \text {. }
$$

Proof. Since any cone $\sigma \in \Sigma$ containing $\rho$ can be written as $\sigma=\rho \oplus \tau$, it follows that $\Sigma$ has a local product structure at $\rho$. Moreover, since the representation of sections of $\mathcal{L}_{\Sigma}$ is distinguished, generators of $\mathcal{L}_{\sigma}$ can be chosen as functions on $\tau$ pulled back by the projection $\sigma \rightarrow \tau$ from $\operatorname{Span}(\rho)$. Thus,

$$
\mathcal{L}_{\sigma}=\mathcal{L}_{\tau} \otimes_{B} A
$$

where $B$ is the space of polynomial functions on $V / \operatorname{Span}(\rho)$ and $\mathcal{L}_{\tau}$ is a $B$ module of functions on $\tau$. Letting $M$ be the set of maximal cones in $\operatorname{Link}_{\Sigma} \rho$, we get as in the proof of Lemma 3.4 that $\mathcal{L}(\Delta)$ is the kernel of the map

$$
\left(\bigoplus_{\tau \in M} \mathcal{L}_{\tau} \longrightarrow \bigoplus_{\tau_{1}, \tau_{2} \in M} \mathcal{L}_{\tau_{1} \cap \tau_{2}}\right) \otimes_{B} A
$$

hence a set of $B$-module generators of the kernel of the map in the parentheses forms a set of generators for the $A$-module $\mathcal{L}(\Delta)$.

Lemma 4.5. Let $s=s_{N} \circ \ldots \circ s_{1}$ be a distinguished subdivision of a fan $\Sigma$. Assume that we have an embedding

$$
\mathcal{L}_{\Sigma_{N-1}} \subset\left(s_{N-1} \circ \ldots \circ s_{1}\right)_{*} \mathcal{A}_{\Sigma_{0}}
$$

giving a distinguished representation of sections of $\mathcal{L}_{\Sigma_{N-1}}$ as conewise polynomial functions on the fan $\Sigma_{0}$. Then there exists a primitive embedding

$$
\mathcal{L}_{\Sigma} \subset\left(s_{N}\right)_{*} \mathcal{L}_{\Sigma_{N-1}}
$$

such that the induced representation of sections of $\mathcal{L}_{\Sigma}$ as functions on $\Sigma_{0}$ is distinguished. 
Proof. Note that to construct an embedding $\mathcal{L}_{\Sigma} \subset\left(s_{N}\right)_{*} \mathcal{L}_{\Sigma_{N-1}}$, we have to choose a subsheaf of $\left(s_{N}\right)_{*} \mathcal{L}_{\Sigma_{N-1}}$ satisfying the conditions for being an equivariant intersection cohomology sheaf. This subsheaf is then isomorphic to $\mathcal{L}_{\Sigma}$. Also, it suffices to construct the embedding of the sheaves when restricted to the subfan $\Sigma^{s}$, so we may assume that $\Sigma=\Sigma^{s}$ and $s_{N}: \Sigma_{N-1} \rightarrow \Sigma$ is the star subdivision at $\mathbb{R}_{\geq 0} v$, where $v$ lies in the relative interior of a maximal cone $\sigma$. For any cone $\tau \in \Sigma, \tau \neq \sigma$, we set $\mathcal{L}_{\tau}=\left(\left(s_{N}\right)_{*} \mathcal{L}_{\Sigma_{N-1}}\right)_{\tau}$. If $\tau=\sigma$, then by the previous lemma, generators of $\left(\left(s_{N}\right)_{*} \mathcal{L}_{\Sigma_{N-1}}\right)_{\sigma}=\mathcal{L}([\hat{\sigma}])$ can be chosen as functions on $\partial \sigma$, pulled back by the projection map $\pi: \operatorname{Span} \sigma \rightarrow \operatorname{Span} \sigma / \mathbb{R} v$. We choose a subset of these generators, representing a basis for the primitive part of $\overline{\mathcal{L}([\hat{\sigma}])}$ with respect to a conewise linear function $\lambda$ as in Definition 4.1. Such a subset generates the module $\mathcal{L}_{\sigma}$.

Definition 4.6. Consider the following data associated to a fan $\Sigma$ :

(a) A distinguished subdivision

$$
\Sigma_{0} \stackrel{s_{1}}{\longrightarrow} \Sigma_{1} \stackrel{s_{2}}{\longrightarrow} \ldots \stackrel{s_{N}}{\longrightarrow} \Sigma_{N}=\Sigma
$$

of the fan $\Sigma$.

(b) A sequence of primitive embeddings

$$
e_{1}: \mathcal{L}_{\Sigma_{1}} \subset\left(s_{1}\right)_{*} \mathcal{A}_{\Sigma_{0}}, \quad e_{i}: \mathcal{L}_{\Sigma_{i}} \subset\left(s_{i}\right)_{*} \mathcal{L}_{\Sigma_{i-1}}, \quad i=2, \ldots, N,
$$

such that for any $i=1, \ldots, N$, the induced representation of sections of $\mathcal{L}_{\Sigma_{i}}$ as conewise polynomial functions on the fan $\Sigma_{0}$ is distinguished.

We call such data $\left(\left\{s_{i}\right\},\left\{e_{i}\right\}\right)$ a distinguished pair on $\Sigma$.

The following properties of distinguished pairs are straight-forward:

(1) Every fan $\Sigma$ has a distinguished pair. It suffices to take a barycentric subdivision of $\Sigma$ and construct the primitive embeddings by induction on $i$ as in Lemma 4.5.

(2) Given a distinguished pair on $\Sigma=\Sigma_{N}$, we get a distinguished pair on $\Sigma_{i}$ for any $i=1, \ldots, N-1$.

(3) A distinguished pair on $\Sigma$ can be restricted to a distinguished pair on a subfan $\Delta \subset \Sigma$. We take $\Delta^{s}=\Delta \cap \Sigma^{s}$ and restrict the subdivisions and the sheaves.

(4) If $\Phi: \Delta \rightarrow \Sigma$ is a conewise linear isomorphism, then a distinguished pair on $\Sigma$ can be pulled back to a distinguished pair on $\Delta$.

As an application of these properties, consider a fan $\Sigma$ with a distinguished pair on it and a cone $\rho \in \Sigma$ such that $\rho \cap \Sigma^{s}=\{0\}$. Then $\Sigma$ has a local product structure at $\rho$ :

$$
\overline{\operatorname{Star}}_{\Sigma} \rho=\Lambda \times_{\phi}[\rho] .
$$

We claim that the distinguished pair on $\Sigma$ induces a distinguished pair on $\Lambda$. Indeed, by restriction we get a distinguished pair on $\operatorname{Link}_{\Sigma} \rho \subset \Sigma$, and then we pull this back to $\Lambda$ by the conewise linear isomorphism $\Lambda \rightarrow \operatorname{Link}_{\Sigma} \rho$.

4.3. Poincaré duality. Let $\Sigma$ be a quasi-convex fan. The Poincaré duality pairing

$$
I H^{n-k}(\Sigma) \times I H^{n+k}(\Sigma, \partial \Sigma) \longrightarrow \mathbb{R}
$$


in [1] is constructed by representing sections of $\mathcal{L}(\Sigma)$ as conewise polynomial functions on a barycentric subdivision of $\Sigma$, and then multiplying these functions. Now suppose we have a barycentric subdivision of $\Sigma$ :

$$
\Sigma_{0} \stackrel{s_{0}}{\longrightarrow} \Sigma_{1} \stackrel{s_{1}}{\longrightarrow} \ldots \longrightarrow \Sigma_{N-1} \stackrel{s_{N-1}}{\longrightarrow} \Sigma_{N}=\Sigma .
$$

If we follow [1], to construct the Poincaré duality paring on the fan $\Sigma_{N-1}$, we would have to consider its barycentric subdivision. Instead, we want to represent sections of $\mathcal{L}_{\Sigma_{N-1}}$ as conewise polynomial functions on the fan $\Sigma_{0}$. The proof of Poincaré duality in [1] also works in this case. We indicate only what modifications have to be made.

Let us assume that we have a distinguished pair on $\Sigma$. Thus we represent sections of $\mathcal{L}_{\Sigma}$ as conewise polynomial functions on $\Sigma_{0}$. We claim that multiplication of these functions followed by the evaluation map $I H^{n}\left(\Sigma_{0}\right) \rightarrow \mathbb{R}$ induces a nondegenerate pairing

$$
I H^{n-k}(\Sigma) \times I H^{n+k}(\Sigma, \partial \Sigma) \longrightarrow \mathbb{R}
$$

for any $k \in \mathbb{Z}$.

The proof in [1] has two parts. First, assuming that the statement holds for fans $[\sigma]$ where $\sigma$ is an $n$-dimensional cone, it is shown to hold for any quasi-convex fan of dimension $n$. This part of the proof also works in our case because it does not depend on the representation of sections of $\mathcal{L}_{\Sigma}$ as functions. The second part of the proof deduces Poincaré duality for fans $[\sigma]$, assuming that it holds for fans of dimension less than $n$. Here is where the representation of sections of $\mathcal{L}$ as functions is important and the proof needs to be modified.

Assume that $\Sigma=[\sigma]$ for a cone $\sigma$. Then $\sigma=\tau \oplus \rho$, where $\tau=\sigma \cap\left|\Sigma^{s}\right|$ and $\rho$ is a simplicial cone. If $\tau=\sigma$, then the sequence of subdivisions is a barycentric subdivision of $[\sigma]$ and the proof of [1] applies. If $\tau \neq \sigma$ then since the representation of sections is distinguished, we have the identifications

$$
\begin{aligned}
\mathcal{L}_{\sigma} & =\mathcal{L}_{\tau} \otimes \mathcal{A}_{\rho}, \\
\mathcal{L}([\sigma], \partial \sigma) & =\mathcal{L}([\tau], \partial \tau) \otimes \mathcal{A}([\rho], \partial \rho) .
\end{aligned}
$$

By induction on the dimension we may assume that the Poincaré duality pairing is nondegenerate on $[\tau]$ and $[\rho]$, hence it is nondegenerate on $[\sigma]=[\tau] \times[\rho]$.

\section{Restriction to FACETS}

In [7] McMullen proved that for simplicial projective fans the Hodge-RiemannMinkowski relations in dimension $n-1$ imply the Hard Lefschetz theorem in dimension $n$. The proof, written in the language of polytope algebras, relies on restricting an element of the polytope algebra to the facets of a polytope. We explain this idea in terms of fans and then give a partial generalization to nonsimplicial fans.

We start with a more general situation. Let $\Sigma$ be a complete fan with a distinguished pair. Let $\mathbb{R}_{\geq 0} v \in \Sigma$ be a 1-dimensional cone such that $\mathbb{R}_{\geq 0} v \notin \Sigma^{s}$, and let $\Delta=\overline{\operatorname{Star}}_{\Sigma}\left(\mathbb{R}_{\geq 0} v\right)$. Then $\Sigma$ has a local product structure at $\mathbb{R}_{\geq 0} v$ :

$$
\Delta=\Lambda \times_{\phi}\left[\mathbb{R}_{\geq 0} v\right] .
$$


We let $\pi: \Delta \rightarrow \Lambda$ be the projection, inducing an isomorphism

$$
I H(\Delta) \simeq I H(\Lambda)
$$

For $h \in I H(\Sigma)$, we denote by $\left.h\right|_{\Lambda}$ the image of $h$ under the restriction map $I H(\Sigma) \rightarrow I H(\Delta) \simeq I H(\Lambda)$. In terms of sections, the restriction of $h$ to $\Delta$ can be represented by a function of the form $\pi^{*}(f)$ (see Lemma 4.4); then $f$ represents $\left.h\right|_{\Lambda}$. Note also that the distinguished pair on $\Sigma$ induces a distinguished pair on $\Lambda$, thus we get the non-degenerate Poincaré pairing, and by induction on dimension, the Hodge-Riemann-Minkowski relations associated with a strictly convex conewise linear function on $\Lambda$.

Let $\lambda \in \mathcal{A}^{2}(\Sigma)$ be a conewise linear function supported on $\Delta$, such that $\lambda(v)>0$. Multiplication with $\lambda$ defines a degree 2 map $I H(\Sigma) \rightarrow I H(\Sigma)$, with image lying in $I H(\Delta, \partial \Delta)$.

Lemma 5.1. For any $h, g \in I H(\Sigma)$ we have

$$
\langle\lambda h \cdot g\rangle_{\Sigma}=\left\langle\left.\left. h\right|_{\Lambda} \cdot g\right|_{\Lambda}\right\rangle_{\Lambda}
$$

up to a positive constant factor.

Proof. By assumption, we have a simplicial subdivision $\Sigma_{0}$ of $\Sigma$ such that cohomology classes are represented by conewise polynomial functions on $\Sigma_{0}$. Recall the construction of the evaluation map in the top degree cohomology. When constructing the functions $\Phi_{\sigma}$ for $\sigma \in \Sigma_{0}$ an $n$-dimensional cone such that $\mathbb{R}_{\geq 0} v \subset \sigma$, we set $H_{\sigma, 1}=\left.\lambda\right|_{\sigma}$. Then for any function $f$ on $\Lambda$ we have

$$
\left\langle\lambda \pi^{*}(f)\right\rangle_{\Sigma}=\langle f\rangle_{\Lambda}
$$

up to a constant factor which depends on the metrics on $\Lambda^{n} V^{*}$ and $\Lambda^{n-1}(V / \mathbb{R} v)^{*}$.

We will assume that the constant in the previous lemma is always 1. For later use we also prove

Lemma 5.2. Multiplication with $\lambda$ defines a degree 2 linear map $\lambda: I H(\Sigma) \rightarrow$ $I H(\Delta, \partial \Delta)$. This map factors through the restriction map $I H(\Sigma) \rightarrow I H(\Delta)$ and the map $\left.\lambda\right|_{\Delta}: I H(\Delta) \rightarrow I H(\Delta, \partial \Delta)$ is a degree 2 isomorphism.

Proof. Since $\lambda$ has support on $\Delta$, the only statements that needs a proof is that $\left.\lambda\right|_{\Delta}$ defines an isomorphism. Consider the bilinear form on $I H(\Delta)$

$$
(h, g) \longrightarrow\left\langle\left.\lambda\right|_{\Delta} h \cdot g\right\rangle_{\Sigma}=\left\langle\left.\left. h\right|_{\Lambda} \cdot g\right|_{\Lambda}\right\rangle_{\Lambda} .
$$

The right hand side is the Poincaré pairing on $\Lambda$, hence the map $\left.\lambda\right|_{\Delta}: I H(\Delta) \rightarrow$ $I H(\Delta, \partial \Delta)$ is injective and the image can be identified with the dual of $I H(\Delta)$. By Poincaré duality applied to the fan $\Delta, I H(\Delta, \partial \Delta)$ is the dual of $\operatorname{IH}(\Delta)$, hence the map is an isomorphism.

Let us now return to McMullen's argument. Assume that $\Sigma$ is a simplicial fan, and let $l \in \mathcal{A}^{2}(\Sigma)$ be a strictly convex function, positive on $V-\{0\}$. For each 1-dimensional cone $\mathbb{R}_{\geq 0} v_{i} \in \Sigma$ we let $\lambda_{i} \in \mathcal{A}^{2}(\Sigma)$ be supported on $\Delta_{i}=\overline{\operatorname{Star}}_{\Sigma}\left(\mathbb{R}_{\geq 0} v_{i}\right)$ so that

$$
l=\sum_{i} \lambda_{i}
$$

Associated with $\Delta_{i}$ we have the fan $\Lambda_{i}$ and the projection map $\pi_{i}$ as before. 
Suppose there exists an $h \in I H^{n-k}(\Sigma)$ such that

$$
l^{k} \cdot h=0 .
$$

We restrict this equality to $\Lambda_{i}$. Note that we may change $l$ by a global linear function so that $\left.l\right|_{\Delta_{i}}=\pi_{i}^{*}\left(l_{i}\right)$ for some strictly convex function $l_{i}$ on $\Lambda_{i}$. It follows that

$$
\left.h\right|_{\Lambda_{i}} \in I P_{l_{i}}\left(\Lambda_{i}\right) .
$$

If we assume the Hodge-Riemann-Minkowski relations in dimension $n-1$, we get

$$
(-1)^{\frac{n-k}{2}}\left\langle\left.\left. l_{i}^{k-1} h\right|_{\Lambda_{i}} \cdot h\right|_{\Lambda_{i}}\right\rangle_{\Lambda_{i}}=(-1)^{\frac{n-k}{2}}\left\langle\lambda_{i} l^{k-1} h \cdot h\right\rangle_{\Sigma} \geq 0,
$$

and the equality holds if and only if $\left.h\right|_{\Lambda_{i}}=0$. Summing over all $i$,

$$
\sum_{i}(-1)^{\frac{n-k}{2}}\left\langle\lambda_{i} l^{k-1} h \cdot h\right\rangle_{\Sigma}=(-1)^{\frac{n-k}{2}}\left\langle l^{k} h \cdot h\right\rangle_{\Sigma} .
$$

Since $l^{k} h=0$, we get that $\left.h\right|_{\Lambda_{i}}=0$ for all $i$.

To finish McMullen's proof, it suffices to show that if $\left.h\right|_{\Lambda_{i}}=0$ for all $i$ then $h=0$. This can be done by showing that $\mathcal{A}(\Sigma)$ is generated as an $A$ module by elements having support on $\Delta_{i}$; then the Poincaré pairing between $h$ and any such element is zero. An explicit set of generators supported on $\Delta_{i}$ was constructed by Timorin in [10.

In the nonsimplicial case we have the following partial generalization of this argument. If $\Sigma$ has a distinguished pair on it and $\mathbb{R}_{\geq 0} v_{i} \in \Sigma$ is a 1-dimensional cone such that $\mathbb{R}_{\geq 0} v_{i} \notin \Sigma^{s}$, then $\Sigma$ has a local product structure at $\mathbb{R}_{\geq 0} v_{i}$. We use the same notation $\Delta_{i}, \Lambda_{i}, \pi_{i}, l_{i}$ associated with the ray $\mathbb{R}_{\geq 0} v_{i}$ as in the simplicial case.

Lemma 5.3. Let $\Sigma$ be a complete $n$-dimensional fan with a distinguished pair on it, such that $\Sigma^{s} \subset[\sigma]$ for a single cone $\sigma \in \Sigma$, and let $l \in \mathcal{A}^{2}(\Sigma)$ be a strictly convex function. Then the Hodge-Riemann-Minkowski relations in dimension $n-1$, imply that any $h \in I H^{n-k}(\Sigma)$ such that $l^{k} h=0$ must satisfy

$$
\left.h\right|_{\Lambda_{i}}=0
$$

for all $i$ such that $\mathbb{R}_{\geq 0} v_{i} \in \Sigma-[\sigma]$.

Proof. Let $\mathbb{R}_{\geq 0} v_{i} \in \Sigma-[\sigma]$ be a 1 -dimensional cone. Restricting the equality $l^{k} h=0$ to $\Lambda_{i}$, we get that $\left.h\right|_{\Lambda_{i}} \in I P_{l_{i}}\left(\Lambda_{i}\right)$.

Now we modify the function $l$ by a global linear function so that $\left.l\right|_{\sigma}=0$, $l(x)>0$ for $x \notin \sigma$, and write

$$
l=\sum_{\mathbb{R}_{\geq 0} v_{i} \in \Sigma-[\sigma]} \lambda_{i}
$$

with $\lambda_{i}$ as before. Then

$$
\begin{gathered}
0=(-1)^{\frac{n-k}{2}}\left\langle l^{k} h \cdot h\right\rangle_{\Sigma}=\sum_{\mathbb{R}_{\geq 0} v_{i} \in \Sigma-[\sigma]}(-1)^{\frac{n-k}{2}}\left\langle\lambda_{i} l^{k-1} h \cdot h\right\rangle_{\Sigma} \\
=\sum_{\mathbb{R}_{\geq 0} v_{i} \in \Sigma-[\sigma]}\left\langle\left.\left. l_{i}^{k-1} h\right|_{\Lambda_{i}} \cdot h\right|_{\Lambda_{i}}\right\rangle_{\Lambda_{i}} .
\end{gathered}
$$

The primitivity of $\left.h\right|_{\Lambda_{i}}$ implies that all these restrictions must be zero. 


\section{Proof of the MAIN THEOREM}

We now give a proof of Theorem 0.2 .

Let $\Sigma$ be a complete fan with a distinguished pair, and let $l$ be a conewise linear strictly convex function on $\Sigma$. Let

$$
\Sigma_{0} \stackrel{s_{1}}{\longrightarrow} \Sigma_{1} \stackrel{s_{2}}{\longrightarrow} \ldots \stackrel{s_{N}}{\longrightarrow} \Sigma_{N}=\Sigma
$$

be the distinguished subdivision of $\Sigma$, where $s_{i}$ is the star subdivision at the ray $\mathbb{R}_{\geq 0} v_{i}$. We choose for $i=N-1, \ldots, 0$ a strictly convex conewise linear function $l_{i}$ on $\Sigma_{i}$ such that $l_{i}-l_{i+1}$ is supported on $\operatorname{Star}_{\Sigma_{i}} v_{i+1}, l_{N}=l$. Since for all $i=0, \ldots, N$ we have a distinguished pair on the fan $\Sigma_{i}$, we can define the quadratic form $Q_{l_{i}}$ on $I H\left(\Sigma_{i}\right)$. We prove by induction on $i$ that $Q_{l_{i}}$ satisfies the Hodge-Riemann-Minkowski relations on $\operatorname{IH}\left(\Sigma_{i}\right)$. The case $i=0$ follows by our assumption that the relations hold for simplicial fans. The induction step then is to prove the Hodge-Riemann-Minkowski relations for $Q_{l_{N}}=Q_{l}$, assuming that $Q_{l_{N-1}}$ satisfies these relations. We may also assume by induction on the dimension that the Hodge-Riemann-Minkowski relations are satisfied in the case of lower dimensional fans.

To simplify notation, let $\hat{\Sigma} \rightarrow \Sigma$ be a star subdivision at $\mathbb{R}_{\geq 0} v$, where $v$ lies in the relative interior of a cone $\sigma \in \Sigma\left(\hat{\Sigma}=\Sigma_{N-1}\right.$ in the previous notation), and let $\hat{l}$ be a strictly convex conewise linear function on $\hat{\Sigma}$ such that $l-\hat{l}$ is supported on $\operatorname{Star}_{\Sigma} \sigma$.

Let us denote $\Delta=\overline{\operatorname{Star}}_{\Sigma}(\sigma)$ and let $\hat{\Delta} \subset \hat{\Sigma}$ be the star subdivision of $\Delta$ at $\mathbb{R}_{\geq 0} v$. We write

$$
\hat{\Delta}=\Theta \times_{\phi}\left[\mathbb{R}_{\geq 0} v\right]
$$

and let $\pi: \hat{\Delta} \rightarrow \Theta$ be the projection from $\mathbb{R} v$. Changing $l$ and $\hat{l}$ by a linear function if necessary, we may assume that $\hat{l}$ restricted to $\hat{\Delta}$ is the pullback of a strictly convex function $l_{\Theta}$ on $\Theta$.

Next we define an auxiliary fan $\bar{\Delta}$ :

$$
\bar{\Delta}=\Delta \cup \Delta^{\prime}
$$

where

$$
\Delta^{\prime}=\left\{\tau+\mathbb{R}_{\geq 0}(-v) \mid \tau \in \partial \Delta\right\} \cup \partial \Delta .
$$

On $\bar{\Delta}$ we have a conewise linear function $\bar{l}$ :

$$
\bar{l}(x)= \begin{cases}l(x) & \text { if } x \in|\Delta|, \\ l_{\Theta}(\pi(x)) & \text { if } x \in\left|\Delta^{\prime}\right| .\end{cases}
$$

Then $\bar{l}$ is strictly convex on $\bar{\Delta}$. Indeed, it suffices to prove that $\bar{l}(x)>l_{\Theta}(\pi(x))$ for $x$ in the interior of $|\Delta|$; this follows from the inequality $l(x)>\hat{l}(x)$ for $x$ in the interior of $|\Delta|$.

We claim that the distinguished pair on $\Sigma$ naturally induces a distinguished pair on $\bar{\Delta}$. The restriction of the distinguished pair on $\Sigma$ to $\Delta$ has $\Delta^{s}=[\sigma]$, thus we get a distinguished subdivision of $\bar{\Delta}$ by setting $\bar{\Delta}^{s}=[\sigma]$ with the same centers of subdivisions as in $\Delta$. Recall that to define the second component of a distinguished pair, it suffices to construct the embeddings of sheaves over the subfan $\bar{\Delta}^{s}=[\sigma]$. For this we take the same embeddings as in $\Delta$. 
Given the distinguished pair on $\bar{\Delta}$, we get the Poincaré duality pairing and the quadratic form $Q_{\bar{l}}$ on $I H(\bar{\Delta})$. We define the evaluation maps on $I H^{2 n}(\Sigma)$ and $I H^{2 n}(\bar{\Delta})$ using the same metric on $\Lambda^{n} V^{*}$.

By the choice of the distinguished pairs on $\Sigma$ and $\bar{\Delta}$, we have an identification $\mathcal{L}_{\Sigma}(\Delta)=\mathcal{L}_{\bar{\Delta}}(\Delta)$. Denote by $F$ the $A$-module

$$
F=\left\{\left(s_{1}, s_{2}\right) \in \mathcal{L}(\Sigma) \times \mathcal{L}(\bar{\Delta})\left|s_{1}\right|_{\Delta}=\left.s_{2}\right|_{\Delta}\right\} .
$$

On $F$ we define the degree 2 map $l_{F}=(l, \bar{l})$, and the quadratic form

$$
Q_{F}\left(\left(s_{1}, s_{2}\right)\right)=Q_{l}\left(s_{1}\right)-Q_{\bar{l}}\left(s_{2}\right) .
$$

It is clear that $Q_{F}$ descends to a quadratic form on the quotient $\bar{F}=F / I F$.

Lemma 6.1. There exists a morphism of A-modules

$$
\beta: F \longrightarrow \mathcal{L}(\hat{\Sigma}),
$$

satisfying

(a) $\beta \circ l_{F}=\hat{l} \circ \beta$;

(b) $Q_{F}=Q_{\hat{\imath}} \circ \beta$.

Proof. Let $B=\operatorname{Sym} W^{*}$, where $V=W \oplus \mathbb{R} v$ and $\Theta$ is a complete fan in $W$. Using Lemma 3.4 we have an isomorphism of $A$-modules $\alpha: \mathcal{L}\left(\Delta^{\prime}\right) \rightarrow \mathcal{L}(\hat{\Delta})$ given as a composition:

$$
\mathcal{L}\left(\Delta^{\prime}\right) \simeq \mathcal{L}(\Theta) \otimes_{B} A \simeq \mathcal{L}(\hat{\Delta}) .
$$

This isomorphism commutes with the actions of $\bar{l}$ and $\hat{l}$ because both functions are defined as pullbacks of $l_{\Theta}$. By Lemma 4.4, the isomorphism $\alpha$ is also compatible with the representations of sections of $\mathcal{L}$ as functions.

Define $\beta$ as follows. For $s=\left(s_{1}, s_{2}\right) \in F$, set

$$
\left.\beta(s)\right|_{\hat{\Sigma}-\hat{\Delta}}=\left.s_{1}\right|_{\Sigma-\Delta},\left.\quad \beta(s)\right|_{\hat{\Delta}}=\alpha\left(\left.s_{2}\right|_{\Delta^{\prime}}\right) .
$$

Since the restriction maps and $\alpha$ are $A$-linear, so is $\beta$. The properties $(a)$ and (b) follow easily from this construction. We comment only on the minus sign in the expression of $Q_{F}$. In Brion's construction of the evaluation map, if $\sigma_{1} \in \hat{\Delta}$ and $\sigma_{2} \in \Delta^{\prime}$ are maximal cones that share a common facet, then the functions $\Phi_{\sigma_{1}}$ and $\Phi_{\sigma_{2}}$ differ by a minus sign. Hence, $\alpha$ introduces a minus sign into the evaluation map.

We use the isomorphism $\alpha: \mathcal{L}\left(\Delta^{\prime}\right) \rightarrow \mathcal{L}(\hat{\Delta})$ defined in the proof of the previous lemma to construct a splitting of the exact sequence

$$
0 \longrightarrow \mathcal{L}\left(\Delta^{\prime}, \partial \Delta^{\prime}\right) \longrightarrow \mathcal{L}(\bar{\Delta}) \longrightarrow \mathcal{L}(\Delta) \longrightarrow 0 .
$$

If $s \in \mathcal{L}(\Delta)$, we define $t \in \mathcal{L}(\bar{\Delta})$ so that $\left.t\right|_{\Delta}=s$ and $\left.t\right|_{\Delta^{\prime}}=\alpha^{-1}\left(s^{\prime}\right)$, where $s^{\prime}$ is the image of $s$ under the embedding $\mathcal{L}(\Delta) \subset \mathcal{L}(\hat{\Delta})$. Thus we get

$$
\mathcal{L}(\bar{\Delta}) \simeq \mathcal{L}(\Delta) \oplus \mathcal{L}\left(\Delta^{\prime}, \partial \Delta^{\prime}\right) .
$$

Lemma 6.2. Let $s_{1} \in \mathcal{L}^{n-k}(\Sigma)$ be such that its image $\left[s_{1}\right] \in I H^{n-k}(\Sigma)$ is l-primitive. Then there exists an $s_{2} \in \mathcal{L}^{n-k}(\bar{\Delta})$ satisfying:

(a) $\left(s_{1}, s_{2}\right) \in F$;

(b) the image $\left[s_{2}\right] \in I H^{n-k}(\bar{\Delta})$ is $\bar{l}$-primitive. 
Proof. By Lemma 5.2 we have an isomorphism

$$
I H\left(\Delta^{\prime}, \partial \Delta^{\prime}\right) \simeq I H(\Theta)[-2]
$$

commuting with the action of $\left.\bar{l}\right|_{\Delta^{\prime}}=\pi^{*}\left(l_{\Theta}\right)$ and $l_{\Theta}$. By induction assumption, $l_{\Theta}$ defines a Lefschetz operation on $I H(\Theta)$ centered at degree $n-1$, hence $\bar{l}$ defines a Lefschetz operation on $I H\left(\Delta^{\prime}, \partial \Delta^{\prime}\right)$ centered at degree $n+1$.

Using the splitting

$$
\mathcal{L}(\bar{\Delta}) \simeq \mathcal{L}(\Delta) \oplus \mathcal{L}\left(\Delta^{\prime}, \partial \Delta^{\prime}\right)
$$

we consider $\left.s_{1}\right|_{\Delta}$ as an element in $\mathcal{L}(\bar{\Delta})$. Since the image of $l^{k+1} s_{1}$ in $I H(\Sigma)$ is zero, we get that the image of $\bar{l}^{k+1} s_{1}$ in $I H(\bar{\Delta})$ lies in the summand $I H^{n+k+2}\left(\Delta^{\prime}, \partial \Delta^{\prime}\right)$. By the previous paragraph,

$$
\bar{l}^{k+1}: I H^{n+1-k-1}\left(\Delta^{\prime}, \partial \Delta^{\prime}\right) \longrightarrow I H^{n+1+k+1}\left(\Delta^{\prime}, \partial \Delta^{\prime}\right)
$$

is an isomorphism, hence there exists an element $s_{0} \in \mathcal{L}^{n-k}\left(\Delta^{\prime}, \partial \Delta^{\prime}\right)$, such that the image of $\bar{l}^{k+1}\left(\left.s_{1}\right|_{\Delta}+s_{0}\right)$ in $I H(\bar{\Delta})$ is zero. Thus $s_{2}=\left.s_{1}\right|_{\Delta}+s_{0}$ is the required section.

By the previous two lemmas we are reduced to proving that $Q_{\bar{l}}$ satisfies the Hodge-Riemann-Minkowski relations on $I H(\bar{\Delta})$. Indeed, given a primitive $\left[s_{1}\right] \in I H^{n-k}(\Sigma)$, by Lemma 6.2 there exists a primitive $\left[s_{2}\right] \in I H^{n-k}(\bar{\Delta})$, such that $\left(s_{1}, s_{2}\right) \in F^{n-k}$. By Lemma 6.1 (a) the class $\left[\beta\left(s_{1}, s_{2}\right)\right] \in I H^{n-k}(\hat{\Delta})$ is $\hat{l}$-primitive, and by part $(b)$ of the same lemma, we have

$$
(-1)^{\frac{n-k}{2}} Q_{l}\left(\left[s_{1}\right]\right)=(-1)^{\frac{n-k}{2}} Q_{\hat{l}}\left(\left[\beta\left(s_{1}, s_{2}\right)\right]\right)+(-1)^{\frac{n-k}{2}} Q_{\bar{l}}\left(\left[s_{2}\right]\right) .
$$

The first summand on the right hand side is non-negative by induction assumption, the second one is nonnegative if we know that $Q_{\bar{l}}$ satisfies the HodgeRiemann-Minkowski relations, hence the left hand side is nonnegative. It also follows that the sum is zero if and only if both $\left[\beta\left(s_{1}, s_{2}\right)\right]$ and $\left[s_{2}\right]$ are zero; this implies that in $\bar{F}$ we have $\left[\left(s_{1}, s_{2}\right)\right]=\left[\left(s_{1}^{\prime}, 0\right)\right]$ for some $s_{1}^{\prime}$ such that the class $\left[s_{1}^{\prime}\right] \in I H(\Sigma) \subset I H(\hat{\Sigma})$ is zero, hence $\left[s_{1}\right]=\left[s_{1}^{\prime}\right]=0$.

Lemma 6.3. The map $\bar{l}$ defines a Lefschetz operation on $I H(\bar{\Delta})$ centered at degree $n$.

Proof. By Poincaré duality it suffices to prove that $\bar{l}^{k}: I H^{n-k}(\bar{\Delta}) \rightarrow$ $I H^{n+k}(\bar{\Delta})$ is injective for $k>0$. If $h$ lies in the kernel of this map then by Lemma 5.3 the restriction of $h$ to $I H\left(\Delta^{\prime}\right)$ is zero. Considering the exact sequence

$$
0 \longrightarrow I H(\Delta, \partial \Delta) \longrightarrow I H(\bar{\Delta}) \longrightarrow I H\left(\Delta^{\prime}\right) \longrightarrow 0,
$$

we have that $h \in I H(\Delta, \partial \Delta)$. Since $\bar{l}$ acts on $I H(\Delta, \partial \Delta)$ by multiplication with $l$, we see that $h$ must lie in the kernel of $l^{k}$. We show in the next lemma that $l^{k}: I H^{n-k}(\Delta) \rightarrow I H^{n+k}(\Delta)$ is surjective, hence dually $l^{k}: I H^{n-k}(\Delta, \partial \Delta) \rightarrow I H^{n+k}(\Delta, \partial \Delta)$ is injective and $h=0$.

Lemma 6.4. The map

$$
l^{k}: I H^{n-k}(\Delta) \longrightarrow I H^{n+k}(\Delta)
$$

is surjective for $k>0$. 
Proof. Let $p$ be the projection from the span of $\sigma$, mapping $\operatorname{Link}_{\Delta}(\sigma)$ isomorphically to a complete fan $\Lambda$ in $W \subset V$. Then we can write

$$
\Delta=\Lambda \times_{\phi}[\sigma]
$$

for a function $\phi: W \rightarrow \operatorname{Span}(\sigma)$. We also have the corresponding conewise linear isomorphism

$$
\Phi: \Lambda \times[\sigma] \longrightarrow \Delta,
$$

inducing an isomorphism of vector spaces

$$
\mathcal{L}(\Delta) \simeq \mathcal{L}(\Lambda) \otimes \mathcal{L}([\sigma])
$$

If we let $A_{\Lambda}$ be the ring of polynomial functions on $W$, then the isomorphism of global sections is an isomorphism of $A_{\Lambda}$ modules. Let $I_{\sigma} \subset \mathcal{A}_{\sigma}$ be the ideal generated by linear functions.

It suffices to show that $I H(\Delta) / l^{k} \cdot I H(\Delta)$ is zero in degrees $n+k$ and higher. If we assume that $\left.l\right|_{\sigma}=0$ then $l$ is the pullback by $p$ of a strictly convex function on $\Lambda$. We have

$$
I H(\Delta) / l^{k} \cdot I H(\Delta)=\mathcal{L}(\Delta) /\left(I, l^{k}\right)=\left(I H(\Lambda) /\left(l^{k}\right) \otimes \mathcal{L}([\sigma])\right) /\left(I_{\sigma}\right),
$$

where a linear function $f \in I_{\sigma}$ acts on the tensor product by multiplication with $f \circ \Phi=1 \otimes f+f \circ \phi \otimes 1$. Now applying the Hard Lefschetz theorem to $\Lambda$, we get that $I H(\Lambda) /\left(l^{k}\right)$ is zero in degrees $\operatorname{dim} \Lambda+k$ and greater. Since $\mathcal{L}([\sigma])=\mathcal{L}_{\sigma}$ has generators in degrees less than $\operatorname{dim} \sigma$, we get that $I H(\Delta) / l^{k} \cdot I H(\Delta)$ is zero in degrees $n=\operatorname{dim} \Lambda+k+\operatorname{dim} \sigma=n+k$ and higher.

We divide the remaining proof that $Q_{\bar{l}}$ satisfies the Hodge-Riemann-Minkowski relations into two cases.

Lemma 6.5. Assume that $\operatorname{dim} \sigma=n$. Then there exists an isomorphism

$$
\gamma: I P_{\bar{l}}(\bar{\Delta}) \longrightarrow I P_{l_{\Theta}}(\Theta),
$$

such that $Q_{l_{\Theta}}(\gamma(p))=Q_{\bar{l}}(p)$ for $p \in I P_{\bar{l}}(\bar{\Delta})$. In particular, $Q_{\bar{l}}$ satisfies the Hodge-Riemann-Minkowski relations.

Proof. Consider the splitting

$$
I H(\bar{\Delta}) \simeq I H(\Delta) \oplus I H\left(\Delta^{\prime}, \partial \Delta^{\prime}\right) .
$$

We have by construction of the primitive embeddings

$$
I H(\Delta)=I H([\sigma]) \simeq I P_{l_{\Theta}}(\Theta) .
$$

We claim that $I H(\Delta)$ is the $\bar{l}$ primitive part of $I H(\bar{\Delta})$, thus defining the isomorphism $\gamma$. To see this, note that

$$
I H\left(\Delta^{\prime}, \partial \Delta^{\prime}\right) \simeq I H(\Theta)[-2],
$$

and this isomorphism commutes with $\bar{l}$ and $l_{\Theta}$, thus no element in $I H\left(\Delta^{\prime}, \partial \Delta^{\prime}\right)$ is primitive. On the other hand, when we modify $\bar{l}$ so that it vanishes on $\sigma$, it is clear that $\bar{l}$ maps $I H(\Delta)$ into $I H\left(\Delta^{\prime}, \partial \Delta^{\prime}\right)$ by the composition:

$$
I H(\Delta) \simeq I P_{l_{\Theta}}(\Theta) \stackrel{\iota[-2]}{\longrightarrow} I H(\Theta)[-2] \simeq I H\left(\Delta^{\prime}, \partial \Delta^{\prime}\right),
$$

where $\iota: I P_{l_{\Theta}}(\Theta) \rightarrow I H(\Theta)$ is the inclusion. This shows that $I H(\Delta)=$ $I P_{\bar{l}}(\bar{\Delta})$. 
For $p \in I H^{n-k}(\Delta) \simeq I P_{l_{\Theta}}(\Theta)$, we represent $p$ by a function $\pi^{*}(f)$. Then

$$
Q_{\bar{l}}(p)=\left\langle\bar{l}^{k} \pi^{*}(f) \cdot \pi^{*}(f)\right\rangle_{\bar{\Delta}}=\left\langle l_{\Theta}^{k-1} f \cdot f\right\rangle_{\Theta}=Q_{l_{\Theta}}(\gamma(p)) .
$$

The middle equality follows from the fact that if $\bar{l}$ vanishes on $\sigma$, then $\bar{l}(-v)>0$ and we can apply Lemma 5.1.

Lemma 6.6. Assume that $\operatorname{dim} \sigma<n$. Then the quadratic form $Q_{\bar{l}}$ satisfies the Hodge-Riemann-Minkowski relations on $\operatorname{IH}(\bar{\Delta})$.

Proof. We write

$$
\bar{\Delta}=\Lambda \times_{\phi} \overline{[\sigma]},
$$

where $\overline{[\sigma]} \subset \bar{\Delta}$ is the subfan lying in the linear span of $\sigma, \Lambda$ is a fan conewise linearly isomorphic to $\operatorname{Link}_{\bar{\Delta}}(\sigma)$, and $\phi$ is an appropriate conewise linear map on $|\Lambda|$. Also let

$$
\Phi: \Lambda \times \overline{[\sigma]} \longrightarrow \bar{\Delta}
$$

be the conewise linear isomorphism constructed from $\phi$.

First we show that, changing $\bar{l}$ by a global linear function if necessary, we may assume that $\Phi^{*} \bar{l}$ is strictly convex on $\Lambda \times \overline{[\sigma]}$. Let us assume that $\bar{l}$ vanishes on $\sigma$, hence $\left.\bar{l}\right|_{\Delta}$ is the pullback by the projection $p: \Delta \rightarrow \Lambda$ of a strictly convex function $l_{\Lambda}$ on $\Lambda$, and we write

$$
\bar{l}=p^{*} l_{\Lambda}+\left(\bar{l}-p^{*} l_{\Lambda}\right),
$$

where $\left(\bar{l}-p^{*} l_{\Lambda}\right)$ is supported on $\Delta^{\prime}$. Note also that the restriction of $\left(\bar{l}-p^{*} l_{\Lambda}\right)$ to $\overline{[\sigma]}$ is strictly convex. Now

$$
\Phi^{*} \bar{l}=\Phi^{*} p^{*} l_{\Lambda}+\Phi^{*}\left(\bar{l}-p^{*} l_{\Lambda}\right),
$$

hence $\Phi^{*} \bar{l}$ is the sum of pullbacks by the two projections of strictly convex functions on $\Lambda$ and on $\overline{[\sigma]}$.

Let us consider a one-parameter family of skew products

$$
\bar{\Delta}_{t}=\Lambda \times_{\phi_{t}} \overline{[\sigma]},
$$

where $\phi_{t}=t \phi$ for $0 \leq t \leq 1$, and the corresponding conewise linear isomorphisms

$$
\Phi_{t}: \Lambda \times \overline{[\sigma]} \longrightarrow \bar{\Delta}_{t} .
$$

Then $\bar{\Delta}_{1}=\bar{\Delta}$ and $\bar{\Delta}_{0}=\Lambda \times \overline{[\sigma]}$. We define the strictly convex function $\bar{l}_{t}$ on $\bar{\Delta}_{t}$ by

$$
\bar{l}_{t}=\left(\Phi_{t}^{-1}\right)^{*} \Phi^{*}(\bar{l}) .
$$

Similarly, we pull back the distinguished pair on $\bar{\Delta}$ to a distinguished pair on $\bar{\Delta}_{t}$ for all $0 \leq t \leq 1$.

Note that the cohomology spaces $I H\left(\bar{\Delta}_{t}\right)$ have the same dimension for all $t \in[0,1]$. By Lemma 6.3 applied to the fan $\bar{\Delta}_{t}$, we also know that $\bar{l}_{t}$ induces a Lefschetz operation on $I H\left(\bar{\Delta}_{t}\right)$, hence the quadratic form $Q_{\bar{l}_{t}}$ has maximal rank. Since all the data defining $Q_{\bar{l}_{+}}$is pulled back from the fan $\bar{\Delta}$, it is clear that the signature of $Q_{\bar{l}_{t}}$ varies continuously with t. Then since $Q_{\bar{l}_{0}}$ has the correct signature by induction on dimension and Corollary 3.3, the same must be true for any $t$. 


\section{REFERENCES}

[1] G. Barthel, J.-P. Brasselet, K.-H. Fieseler and L. Kaup, Combinatorial Intersection cohomology for Fans, Tôhoku Math. J. 54, 2002, 1-41.

[2] P. Bressler and V. A. Lunts, Intersection Cohomology on Nonrational Polytopes, preprint nath.AG/0002006.

[3] M. Brion, The Structure of the Polytope Algebra, Tôhoku Math. J. 49, 1997, 1-32.

[4] K.-H. Fieseler, Rational Intersection Cohomology of Projective Toric Varieties, Journal f.d. reine u. angew. Mathematik 413 (1991), 88-98.

[5] M. Goresky, R. Kottwitz, R. MacPherson, Equivariant Cohomology, Koszul duality and the localization theorem, Invent. math., 131 (1998), 1, 25-83.

[6] P. Griffiths and J. Harris, Principles of Algebraic Geometry, John Wiley \& Sons, Inc. 1978.

[7] P. McMullen, On Simple Polytopes, Invent. math. 113, 419-444 (1993).

[8] R. Stanley, The number of faces of a simplicial convex polytope, Adv. Math. 35, 1980, 236-238.

[9] R. Stanley, Generalized h-vectors, intersection cohomology of toric varieties and related results, in Commutative Algebra and Combinatorics, M. Nagata and H. Matsumura, eds., Adv. Std. Pure Math. 11, Kinokunia, Tokyo, and North Holland, Amsterdam/New York, 1987, 187-213.

[10] V. Timorin, On polytopes simple in edges, (Russian) Funktsional. Anal. i Prilozhen. 35 (2001), no. 3, 36-47, 95; translation in Funct. Anal. Appl. 35 (2001), no. 3, 189-198.

[11] V. Timorin, An analogue of the Hodge-Riemann relations for simple convex polytopes, Russian Math. Surveys 54, No. 2, 1999, 381-426.

Department of Mathematics, Harvard University, 1 Oxford Street, CamBRIDGE, MA 02138, USA

E-mail address: kkaru@math.harvard.edu 